\title{
Doubling up on supersymmetry in the Higgs sector
}

\author{
John Ellis, ${ }^{a, b}$ Jérémie Quevillon ${ }^{a}$ and Verónica Sanz ${ }^{c}$ \\ ${ }^{a}$ Theoretical Particle Physics \& Cosmology Group, Department of Physics, King's College London, \\ Strand, London WC2R 2LS, U.K. \\ ${ }^{b}$ Theoretical Physics Department, CERN, \\ CH 1211 Geneva 23, Switzerland \\ ${ }^{c}$ Department of Physics and Astronomy, University of Sussex, \\ Brighton BN1 9QH, U.K. \\ E-mail: John.Ellis@cern.ch, jeremie.quevillon@kcl.ac.uk, \\ v.sanz@sussex.ac.uk
}

ABSTRACT: We explore the possibility that physics at the $\mathrm{TeV}$ scale possesses approximate $N=2$ supersymmetry, which is reduced to the $N=1$ minimal supersymmetric extension of the Standard Model (MSSM) at the electroweak scale. This doubling of supersymmetry modifies the Higgs sector of the theory, with consequences for the masses, mixings and couplings of the MSSM Higgs bosons, whose phenomenological consequences we explore in this paper. The mass of the lightest neutral Higgs boson $h$ is independent of $\tan \beta$ at the tree level, and the decoupling limit is realized whatever the values of the heavy Higgs boson masses. Radiative corrections to the top quark and stop squarks dominate over those due to particles in $N=2$ gauge multiplets. We assume that these radiative corrections fix $m_{h} \simeq 125 \mathrm{GeV}$, whatever the masses of the other neutral Higgs bosons $H, A$, a scenario that we term the h2MSSM. Since the $H, A$ bosons decouple from the $W$ and $Z$ bosons in the $h 2 \mathrm{MSSM}$ at tree level, only the LHC constraints on $H, A$ and $H^{ \pm}$couplings to fermions are applicable. These and the indirect constraints from LHC measurements of $h$ couplings are consistent with $m_{A} \gtrsim 200 \mathrm{GeV}$ for $\tan \beta \in(2,8)$ in the $h 2 \mathrm{MSSM}$.

KeYWORDS: Supersymmetry Phenomenology

ARXIV EPRINT: 1607.05541 


\section{Contents}

1 Introduction 1

2 The $N=2$ supersymmetric Higgs sector 3

2.1 Model framework 3

2.2 Tree-level analysis 3

2.3 Radiative corrections $\quad 6$

2.4 The stop sector in the $h$ MSSM and the $h 2 \mathrm{MSSM} \quad 10$

3 Constraints from LHC measurements $\quad 11$

3.1 Constraints from $H / A / H^{ \pm}$searches 12

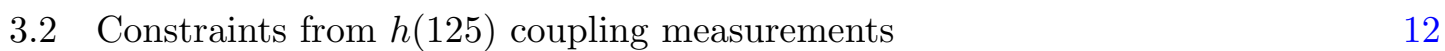

$\begin{array}{lll}3.3 & \text { Constraints from } \Gamma(h \rightarrow g g, \gamma \gamma) & 15\end{array}$

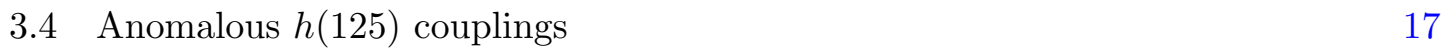

$\begin{array}{llr}4 \text { Conclusions } & 20\end{array}$

\section{Introduction}

Since the Standard Model is chiral, it can accommodate only $N=1$ supersymmetry, as in the minimal supersymmetric extension of the Standard Model (MSSM). On the other hand, any new physics beyond the Standard Model would contain vector-like representations of the $\mathrm{SU}(2) \times \mathrm{U}(1)$ gauge group of the Standard Model. As such, it could accommodate $N=2$ supersymmetry. One could even argue that it should possess the maximum possible degree of supersymmetry, namely $N=2$. Indeed, there are plenty of theoretical set-ups that lead naturally to a chiral $N=1$ supersymmetry model at the electroweak scale with a vector-like $N=2$ extension at the TeV scale, including models invoking extra dimensions and superstring model constructions [1-9].

Studies of possible $N=2$ extensions of the Standard Model have a long history, with considerable attention paid to the gauge and matter sectors of such models. An $N=2$ vector multiplet would contain more degrees of freedom than in the MSSM. In particular, gauginos would no longer be Majorana particles, but Dirac. Moreover, additional adjoint scalar fields would appear, namely a new singlet $S$, triplet $T$ and octet $O$. The phenomenology of the Dirac gauginos has been explored in a number of papers [10-36], and attention also been paid to the Higgs sector of an $N=2$ extension of the Standard Model, which has interesting differences from the Higgs sector of the MSSM [1-4]. This is a natural entry point into phenomenological studies of $N=2$ models, since the Higgs sector of the MSSM is necessarily vector-like, and hence readily modified to realize $N=2$ supersymmetry. Moreover, the exploration of Higgs phenomenology is well underway, with important 
experimental constraints coming from measurements of the $h(125)$ Higgs boson [37] and searches for the heavier MSSM Higgs bosons.

As has been pointed out in previous studies, the $N=2$ version of the tree-level supersymmetric Higgs potential (2.3) contains an extra term $\frac{1}{2}\left(g_{1}^{2}+g_{2}^{2}\right)\left|H_{1} H_{2}\right|^{2}$, which has important phenomenological consequences [1-4]. In particular, the masses of the Higgs bosons are independent of $\tan \beta$ at the tree level, and the rotation from the doublet basis $H_{1}, H_{2}$ to the mass eigenstate basis $h, H$ is trivial, so that at the tree level the $N=2$ model realizes automatically the decoupling limit of the MSSM. Hence the tree-level couplings of the lighter neutral scalar Higgs boson $h$ are necessarily identical to those of a Standard Model Higgs, and the heavier neutral scalar boson $H$ plays no role in electroweak symmetry breaking.

These observations are modified by the radiative corrections to the Higgs sector, of which the most important are those due to the top-stop sector, as in the MSSM. ${ }^{1}$ As in the MSSM, a practical way to analyze Higgs phenomenology in the model with $N=2$ supersymmetry is to use the measured mass of the observed Standard Model-like Higgs boson $m_{h} \simeq 125 \mathrm{GeV}$ as a constraint on the other parameters of the model. In the MSSM case, this has been called the $h$ MSSM scenario: the analogous scenario we propose here is termed the $h 2 \mathrm{MSSM}$ scenario.

As we show, an important difference between the $h$ MSSM and $h 2 \mathrm{MSSM}$ scenarios is that the latter can be realized with smaller stop masses than the former for any value of $m_{A} \gtrsim m_{h}$, and for smaller $m_{A}$ for any fixed values of the stop masses and $\tan \beta$. This observation then raises the question how light the heavier Higgs bosons $H, A$ can be in the h2MSSM, for what range of $\tan \beta$.

The LHC constraints on $H \rightarrow W^{+} W^{-}, Z^{0} Z^{0}$ and $A \rightarrow Z h$ decays are not relevant for the $h 2 \mathrm{MSSM}$, since it realizes automatically the decoupling limit at the tree level, and the $H W^{+} W^{-}$and $H Z^{0} Z^{0}$ couplings induced at the loop level are relatively small. On the other hand, LHC constraints on decays of the heavy Higgs bosons into fermions are in principle relevant. Specifically, the constraint from the search for $H^{ \pm} \rightarrow \tau^{ \pm} \nu$ decays is the same as in the $h$ MSSM. Before saying the same for the LHC constraint on $A / H \rightarrow \tau^{+} \tau^{-}$, one must check the near-degeneracy of the $H$ and $A$, as assumed in the experimental analyses. As we show, in the $h 2 \mathrm{MSSM} m_{H}-m_{A}$ is actually typically significantly smaller in magnitude than in the $h \mathrm{MSSM}$. Consequently, the LHC constraints on $A / H \rightarrow \tau^{+} \tau^{-}$are directly applicable to the $h 2 \mathrm{MSSM}$.

Also, measurements at LHC Run 1 of the couplings of the $h(125)$ to fermions impose important indirect constraints on the $h 2 \mathrm{MSSM}$ in the $\left(m_{A}, \tan \beta\right)$ plane, though they are weaker than in the $h \mathrm{MSSM}$. As we show, the principal constraints are those on the ratios of $h$ couplings to up-type quarks, down-type quarks and massive vector bosons, and that on the $h \gamma \gamma$ coupling. We find that the direct searches for heavy Higgs bosons exclude ranges of $m_{A}$ when $\tan \beta \gtrsim 7$, and the $h$ coupling measurements require $m_{A} \gtrsim 185 \mathrm{GeV}$ in the h2MSSM, compared with $\gtrsim 350 \mathrm{GeV}$ in the $h \mathrm{MSSM}$.

\footnotetext{
${ }^{1}$ There are also tree-level corrections due to the $N=2$ gauge sectors of the theory, but it was found in [38] that the contributions of the additional adjoints $S$ and $T$ to the Higgs boson masses are typically two orders of magnitude smaller than the loop contributions we consider below, for values of $m_{S, T} \sim m_{\tilde{t}}$.
} 
This paper is organized as follows. In section 2, we show the differences between the MSSM and the $N=2$ Higgs sector, at the tree level in section 2.2 and including radiative corrections in section 2.3, and we use the dominant loop corrections from the stop sector in both the $h \mathrm{MSSM}$ and the $h 2 \mathrm{MSSM}$ to evaluate possible stop masses in section 2.4. Constraints from the LHC are studied in section 3, where we discuss the current direct constraints from searches for $H, A$ and $H^{ \pm}$in section 3.1, bounds on the $N=2$ Higgs sector from $h f \bar{f}, h W^{+} W^{-}$and $h Z^{0} Z^{0}$ couplings in section 3.2 and those from the $h \gamma \gamma$ and $h g g$ couplings in section 3.3. We also discuss the sizes of anomalous couplings of $h(125)$ that could be constrained by future measurements in section 3.4. We conclude in section 4 .

\section{The $N=2$ supersymmetric Higgs sector}

\section{$2.1 \quad$ Model framework}

The Lagrangian for an $N=2$ extension of the Standard Model possesses an $R$ symmetry, and its $\mathrm{SU}(2)_{R} \times \mathrm{U}(1)_{R}^{N=2}$-invariant form can be written in the $N=1$ language as $[8,9]$ :

$$
\begin{aligned}
L= & \frac{1}{8 g^{2}}\left[W^{\alpha} W_{\alpha}\right]_{F}+\left[\sqrt{2} i g Y \Phi_{V} X\right]_{F}+\text { h.c. } \\
& +\left[2 \operatorname{Tr}\left(\Phi_{V}^{\dagger} \mathrm{e}^{2 g V} \Phi_{V} \mathrm{e}^{-2 g V}+X^{\dagger} \mathrm{e}^{2 g V} X+Y^{\dagger} \mathrm{e}^{-2 g V^{T}} Y\right)\right]_{D},
\end{aligned}
$$

where $\Phi_{V} \equiv \Phi_{V}^{a} T^{a}$ and $V \equiv V^{a} T^{a}$, where the $T^{a}$ are the gauge group generators. The second $F$-term in the upper line of (2.1) is the superpotential, whose only free parameter is the gauge coupling constant $g$. The coupling constant of the Yukawa term in the superpotential is determined by the gauge coupling due to the $\mathrm{SU}(2)_{R}$ global symmetry. The $\mathrm{SU}(2)_{R}$ symmetry forbids any chiral Yukawa terms, so that fermion mass generation in the $N=2$ sector is linked to supersymmetry breaking. We note also that the $\mathrm{U}(1)_{R}^{N=2}$ symmetry forbids any mass terms of the form $W_{2} \ni \mu^{\prime} X Y$, and specifically that the usual $N=1$ $\mu$ term $W \sim \mu H_{1} H_{2}$ is forbidden by the full $R$-symmetry. A theory with no $\mu$-term would lead to unacceptably light charginos [39-41], but couplings of the Higgs multiplet to the adjoint scalars of an $\mathrm{N}=2$ gauge sector provide mechanisms to lift the chargino masses and additional $\mu$-like contributions to the scalar potential [36]. Note that, unlike the $\mathrm{SU}(2)_{R}$ global symmetry, the $\mathrm{U}(1)_{R}^{N=2}$ symmetry can survive supersymmetry breaking.

Finally, the $N=2$ Higgs sector belongs to a hypermultiplet $\mathbb{H}=\left(\mathcal{H}^{c}, \mathcal{H}\right)$ whose interactions with the gauge sector are given by the Lagrangian

$$
\int d^{4} \theta\left\{\mathcal{H}^{\dagger} e^{V} \mathcal{H}+\mathcal{H}^{c} e^{-V} \mathcal{H}^{c \dagger}\right\}-\left\{\sqrt{2} \int d^{2} \theta \mathcal{H}^{c} \chi \mathcal{H}+\text { h.c. }\right\} .
$$

In the following we analyze the phenomenology of this $N=2$ framework for the Higgs sector of the MSSM.

\subsection{Tree-level analysis}

We can write the tree-level $N=2$ Higgs potential in the usual MSSM notation where $H_{1,2}$ are the lowest components of the chiral superfields $\mathcal{H}$ and $\mathcal{H}^{c}$ respectively. The $\mathrm{H}_{2}$ field 
gives masses to up-type quarks and the $H_{1}$ field gives masses to down-type fermions. The potential for these neutral components of the Higgs doublets is

$$
\begin{aligned}
V= & m_{1}^{2}\left|H_{1}\right|^{2}+m_{2}^{2}\left|H_{2}\right|^{2}-m_{3}^{2}\left(H_{1} H_{2}+h . c .\right) \\
& +\frac{1}{8}\left(g_{1}^{2}+g_{2}^{2}\right)\left(\left|H_{1}\right|^{2}-\left|H_{2}\right|^{2}\right)^{2}+\frac{1}{2}\left(g_{1}^{2}+g_{2}^{2}\right)\left|H_{1} H_{2}\right|^{2},
\end{aligned}
$$

where $m_{i}^{2}=m_{H_{i}}^{2}+\mu^{2}$ are the effective low-energy mass parameters including the soft supersymmetry-breaking and $\mu$ terms. In the last line of $(2.3),{ }^{2}$ the first quartic term is the usual $D$-term of the $N=1 \mathrm{MSSM}$, whereas the second is a specific $N=2$ effect. This extra quartic term in the potential has interesting consequences for the minimization of the potential and the Higgs spectrum, as we now review.

The conditions to have a vacuum that breaks electroweak symmetry with the correct value of $m_{Z}$ for a specific value of $\tan \beta$ are:

$$
\begin{aligned}
\frac{m_{Z}^{2}}{2} & =-\mu^{2}+\frac{1}{\tan ^{2} \beta-1}\left(m_{H_{1}}^{2}-m_{H_{2}}^{2} \tan ^{2} \beta\right), \\
m_{A}^{2} & =m_{H_{1}}^{2}+m_{H_{2}}^{2}+2 \mu^{2}+m_{Z}^{2} .
\end{aligned}
$$

We note the difference between (2.5) and the corresponding MSSM minimization condition $m_{A}^{2}=m_{H_{1}}^{2}+m_{H_{2}}^{2}+2 \mu^{2}$, which has the consequence that the value of $m_{A}$ in the $N=2$ model is larger than that in the MSSM for the same input mass parameters.

In the $\left(H_{1}, H_{2}\right)$ basis for the two Higgs doublet fields, the CP-even $h / H$ mass matrix can be written in terms of the $Z$ and $A$ boson masses and the angle $\beta$. In the MSSM, the tree-level mass-squared matrix is

$$
\mathcal{M}_{\text {tree }}^{2, M S S M}=\left(\begin{array}{cc}
m_{Z}^{2} \cos ^{2} \beta+m_{A}^{2} \sin ^{2} \beta & -\left(m_{A}^{2}+m_{Z}^{2}\right) \cos \beta \sin \beta \\
-\left(m_{A}^{2}+m_{Z}^{2}\right) \cos \beta \sin \beta & m_{Z}^{2} \sin ^{2} \beta+m_{A}^{2} \cos ^{2} \beta
\end{array}\right) .
$$

On the other hand, if the Higgs sector has $N=2$ supersymmetry, the tree-level masssquared matrix is [1-4]:

$$
\mathcal{M}_{\text {tree }}^{2, N 2}=\left(\begin{array}{cc}
m_{Z}^{2} \cos ^{2} \beta+m_{A}^{2} \sin ^{2} \beta & -\left(m_{A}^{2}-m_{Z}^{2}\right) \cos \beta \sin \beta \\
-\left(m_{A}^{2}-m_{Z}^{2}\right) \cos \beta \sin \beta & m_{Z}^{2} \sin ^{2} \beta+m_{A}^{2} \cos ^{2} \beta
\end{array}\right),
$$

where we note the crucial change: $m_{A}^{2}+m_{Z}^{2} \rightarrow m_{A}^{2}-m_{Z}^{2}$ in the off-diagonal terms from the MSSM case (2.6). ${ }^{3}$ The eigenvalues of the matrices (2.6), (2.7) correspond to the physical masses-squared of the neutral CP-even Higgs bosons. In the MSSM case they are

$$
m_{h / H}^{2, M S S M}=\frac{1}{2}\left(m_{A}^{2}+m_{Z}^{2} \mp \sqrt{m_{A}^{4}+m_{Z}^{4}-2 m_{A}^{2} m_{Z}^{2} \cos 4 \beta}\right)
$$

\footnotetext{
${ }^{2}$ On should note that in the absence of Majorana mass from adjoint superfields [10], no low-energy Dterm quartic couplings will be present (including the Higgs quartic terms). As customary, we then assume the presence of Majorana masses, which would lead to non-vanishing quartic couplings. We assume the Bino-Wino masses are of the order of the TeV scale, hence they do not modify substantially the Higgs phenomenology and do not open new decay channels for the Higgs.

${ }^{3}$ We note in passing that there is a missing minus sign in the off-diagonal terms in Equation (3.12) of [1-4].
} 

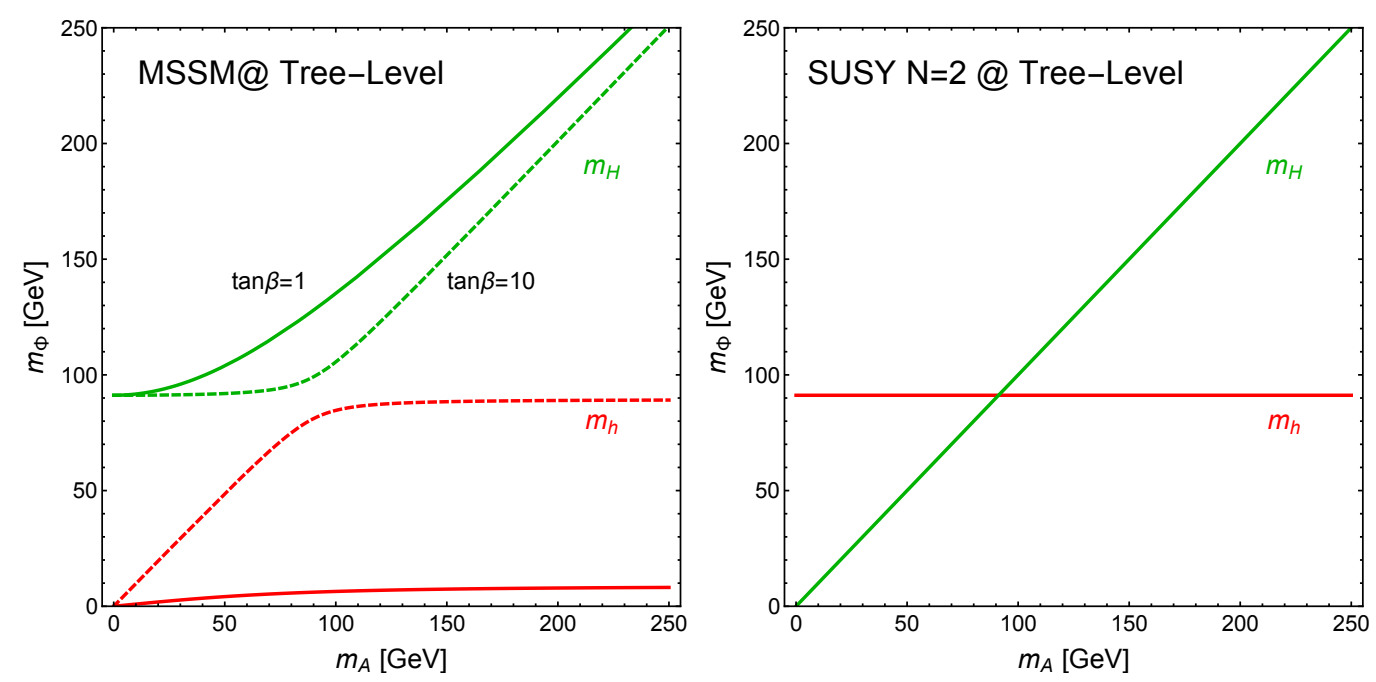

Figure 1. The tree-level CP-even Higgs masses $m_{h}$ (red lines) and $m_{H}$ (green lines) in the MSSM (left panel, for $\tan \beta=1$ (solid lines) and $\tan \beta=10$ (dashed lines)) and for the $N=2$ MSSM (right panel), as functions of the CP-odd Higgs mass $m_{A}$.

and the mass of the charged Higgs boson is

$$
m_{H^{ \pm}}^{M S S M}=\sqrt{m_{A}^{2}+m_{W}^{2}}
$$

at the tree level, ${ }^{4}$ whereas in the $N=2$ case they are

$$
m_{h}^{N 2}=m_{Z} ; \quad m_{H}^{N 2}=m_{A},
$$

and the charged Higgs boson mass is

$$
m_{H^{ \pm}}^{N 2}=\sqrt{m_{A}^{2}+2 m_{W}^{2}} .
$$

We see that, as in the MSSM, the spectrum of the $N=2$ Higgs sector is controlled by $m_{A}$. However, in contrast to the MSSM, it has no dependence on $\tan \beta$ at the tree level.

The left panel of figure 1 shows the tree-level $N=1$ MSSM CP-even neutral Higgs boson masses as functions of $m_{A}$ for different values of $\tan \beta$, and we see that $m_{h}$ increases with $\tan \beta$, its upper limit being $m_{Z}$. The right panel of figure 1 shows the corresponding $N=2$ CP-even neutral Higgs boson masses at the tree level, where we see that $m_{h}=m_{Z}$ independently of $m_{A}$ and $\tan \beta$, and that $m_{H}$ crosses $m_{h}$ without the 'level repulsion' effect seen in the left panel.

The physical CP-even Higgs bosons are obtained from the Higgs doublet fields $\left(H_{1}, H_{2}\right)$ by rotation through an angle $\alpha$ :

$$
\left(\begin{array}{l}
H \\
h
\end{array}\right)=\left(\begin{array}{cc}
\cos \alpha & \sin \alpha \\
-\sin \alpha & \cos \alpha
\end{array}\right)\left(\begin{array}{l}
H_{1} \\
H_{2}
\end{array}\right) .
$$

\footnotetext{
${ }^{4}$ We note also that the supersymmetric radiative corrections to this relation are known to be small in general in this model.
} 
The MSSM mass-squared matrix (2.6) is diagonalized by the following mixing angle:

$$
\alpha_{M S S M}=\frac{1}{2} \arctan \left(\tan 2 \beta \frac{m_{A}^{2}+m_{Z}^{2}}{m_{A}^{2}-m_{Z}^{2}}\right),
$$

which satisfies the relation $-\pi / 2 \leq \alpha \leq 0$. On the other hand, the $N=2$ mass matrix (2.7) is diagonalized by the following mixing angle:

$$
\alpha_{N 2}=\beta-\frac{\pi}{2}
$$

which also satisfies the relation $-\pi / 2 \leq \alpha \leq 0$.

This implies that at the tree level the $N=2$ theory realizes automatically the decoupling limit, in which the lighter CP-even neutral Higgs boson $h$ has Standard Model-like couplings and the heavier one, $H$, does not couple to gauge bosons.

\subsection{Radiative corrections}

In our approach, the Higgs sector is described in terms of just the parameters entering the tree-level expressions for the masses and mixing, supplemented by the experimentallyknown value of $m_{h}$. In this sense, the $h \mathrm{MSSM}$ and $h 2 \mathrm{MSSM}$ approaches can be considered as 'model-independent', as the predictions for the properties of the Higgs bosons do not depend on the details of the unobserved supersymmetric sector. We write the mass matrix for the neutral CP-even states as

$$
\mathcal{M}_{\Phi}^{2}=\mathcal{M}_{\text {tree }}^{2}+\left(\begin{array}{cc}
\Delta \mathcal{M}_{11}^{2} & \Delta \mathcal{M}_{12}^{2} \\
\Delta \mathcal{M}_{12}^{2} & \Delta \mathcal{M}_{22}^{2}
\end{array}\right)
$$

where the tree-level matrix $M_{\text {tree }}^{2}$ is given in (2.6) and (2.7) for the MSSM and its $N=2$ extension, respectively, and the $\Delta \mathcal{M}_{i j}^{2}$ are the radiative corrections.

The importance of radiative corrections is manifested by the experimental measurement $m_{h}=125 \mathrm{GeV}$. The most important quantum corrections $\epsilon$ to the CP-even neutral Higgs masses come from top and stop loops, which alter only the $\Delta \mathcal{M}_{22}^{2}$ element of the mass-squared matrix. In the MSSM we have:

$$
\mathcal{M}_{\Phi}^{2, M S S M}=\left(\begin{array}{cc}
m_{Z}^{2} \cos ^{2} \beta+m_{A}^{2} \sin ^{2} \beta & -\left(m_{A}^{2}+m_{Z}^{2}\right) \cos \beta \sin \beta \\
-\left(m_{A}^{2}+m_{Z}^{2}\right) \cos \beta \sin \beta & m_{Z}^{2} \sin ^{2} \beta+m_{A}^{2} \cos ^{2} \beta+\epsilon_{M S S M}
\end{array}\right),
$$

where $\epsilon_{M S S M}$ depends on the top quark mass, the stop masses through the combination $M_{S U S Y} \equiv \sqrt{m_{\tilde{t}_{1}} m_{\tilde{t}_{2}}}$, and the mixing parameter in the stop mass matrix, $X_{t}$. A useful approximate expression for $\epsilon_{M S S M}$ is:

$$
\epsilon_{M S S M}=\frac{3 m_{t}^{4}}{2 \pi^{2} v^{2}}\left(\ln \frac{M_{S U S Y}^{2}}{m_{t}^{2}}+\frac{X_{t}^{2}}{2 M_{S U S Y}^{2}}\left(1-\frac{X_{t}^{2}}{6 M_{S U S Y}^{2}}\right)\right) .
$$

In general MSSM models, the value of $m_{h}$ is a complicated function of the model parameters, particularly if one takes into account two- and more-loop effects.

Other radiative corrections to the Higgs mass matrix have been studied in [42-46]. Direct analysis of the dominant one-loop contributions from top-stop loops shows that 
the corrections to the $\Delta \mathcal{M}_{11}^{2}$ and $\Delta \mathcal{M}_{12}^{2}$ elements of the CP-even Higgs mass matrix are proportional to powers of the quantity $\mu X_{t} / M_{S U S Y}^{2}$. Consequently, they are negligible to the extent that $\mu X_{t} / M_{S U S Y}^{2} \lesssim 1$.

In MSSM-like scenarios with $M_{S U S Y}$ up to a few $\mathrm{TeV}$, the consideration of the full one-loop contributions or of the known two-loop contributions does not alter this simple picture. ${ }^{5}$ When the SUSY scale is very large, additional checks on the value of $m_{h}$ are required at low $\tan \beta$, for which a comparison with an effective field theory calculation is necessary. Results of such an analysis $[47,48]$ indicate that, even in such heavy- $M_{S U S Y}$ scenarios, the predictions of the $h \mathrm{MSSM}$ agree within a few percent with the exact results for $m_{H}, \alpha$ and $\lambda_{H h h}$, as long as the condition $\mu X_{t} / M_{S U S Y}^{2} \lesssim 1$ is satisfied.

For the purposes of our $N=2$ study here, which is restricted to the Higgs sector, we follow the philosophy proposed in [42-46], in which the $h$ MSSM scenario was introduced to discuss the $N=1 \mathrm{MSSM}$ Higgs sector. The idea is again to use the known output $m_{h}$ instead of the unknown input $\epsilon$, adjusting $\epsilon$ so as to obtain $m_{h}=125 \mathrm{GeV}$. Here we extend this idea to the $N=2$ case, in a scenario we call the $h 2$ MSSM.

In the $N=1$ case, diagonalizing the one-loop corrected mass-squared matrix (2.16) and requiring that one of the eigenvalues of the mass matrix be $m_{h}=125 \mathrm{GeV}$ yields the following simple analytical formula for $\epsilon$ :

$$
\epsilon_{M S S M}=\Delta \mathcal{M}_{22}^{2, M S S M}=\frac{m_{h}^{2}\left(m_{A}^{2}+m_{Z}^{2}-m_{h}^{2}\right)-m_{A}^{2} m_{Z}^{2} \cos ^{2} 2 \beta}{m_{Z}^{2} \cos ^{2} \beta+m_{A}^{2} \sin ^{2} \beta-m_{h}^{2}} .
$$

In this $h \mathrm{MSSM}$ approach the mass of the heavier neutral CP-even $H$ boson and the mixing angle $\alpha$ that diagonalises the $h, H$ states are given by the following simple expressions:

$$
\begin{aligned}
m_{H}^{2, M S S M} & =\frac{\left(m_{A}^{2}+m_{Z}^{2}-m_{h}^{2}\right)\left(m_{Z}^{2} \cos ^{2} \beta+m_{A}^{2} \sin ^{2} \beta\right)-m_{A}^{2} m_{Z}^{2} \cos ^{2} 2 \beta}{m_{Z}^{2} \cos ^{2} \beta+m_{A}^{2} \sin ^{2} \beta-m_{h}^{2}}, \\
\alpha_{M S S M} & =-\arctan \left(\frac{\left(m_{Z}^{2}+m_{A}^{2}\right) \cos \beta \sin \beta}{m_{Z}^{2} \cos ^{2} \beta+m_{A}^{2} \sin ^{2} \beta-m_{h}^{2}}\right),
\end{aligned}
$$

in terms of the inputs $m_{A}, \tan \beta$ and the mass of the lighter CP-even eigenstate $m_{h}=$ $125 \mathrm{GeV}$.

Turning now to the $N=2$ Higgs sector, we can perform the same analysis as before, starting with the mass matrix where the most important quantum corrections $\epsilon$ to the CP-even neutral Higgs masses come from top and stop loops, which alter only the $\Delta \mathcal{M}_{22}^{2}$ element of the mass-squared matrix,

$$
\mathcal{M}_{\Phi}^{2, N 2}=\left(\begin{array}{lc}
m_{Z}^{2} \cos ^{2} \beta+m_{A}^{2} \sin ^{2} \beta & \left(m_{Z}^{2}-m_{A}^{2}\right) \cos \beta \sin \beta \\
\left(m_{Z}^{2}-m_{A}^{2}\right) \cos \beta \sin \beta & m_{Z}^{2} \sin ^{2} \beta+m_{A}^{2} \cos ^{2} \beta+\epsilon_{N 2}
\end{array}\right) .
$$

Requiring $m_{h}^{N 2}=125 \mathrm{GeV}$, we then obtain

$$
\epsilon_{N 2}=\Delta \mathcal{M}_{22}^{2, N 2}=\frac{2\left(m_{A}^{2}-m_{h}^{2}\right)\left(m_{h}^{2}-m_{Z}^{2}\right)}{\cos 2 \beta\left(m_{Z}^{2}-m_{A}^{2}\right)+m_{A}^{2}-2 m_{h}^{2}+m_{Z}^{2}} .
$$

\footnotetext{
${ }^{5}$ For more details about this particular point, the reader should consult references in [46].
} 
The heavier CP-even mass-squared eigenvalue and the rotation angle of the mass matrix are then found to be

$$
\begin{aligned}
m_{H}^{2, N 2} & =m_{A}^{2}-m_{h}^{2}+m_{Z}^{2}+\frac{2\left(m_{A}^{2}-m_{h}^{2}\right)\left(m_{h}^{2}-m_{Z}^{2}\right)}{\cos 2 \beta\left(m_{Z}^{2}-m_{A}^{2}\right)+m_{A}^{2}-2 m_{h}^{2}+m_{Z}^{2}}, \\
\alpha_{N 2} & =-\arctan \left(\frac{\sin 2 \beta\left(m_{A}^{2}-m_{Z}^{2}\right)}{\cos 2 \beta\left(m_{Z}^{2}-m_{A}^{2}\right)+m_{A}^{2}-2 m_{h}^{2}+m_{Z}^{2}}\right) .
\end{aligned}
$$

We note that in both the $h$ MSSM and the $h 2$ MSSM scenarios there is the same minimal value for $m_{A}$ :

$$
m_{A}=\sqrt{\frac{m_{h}^{2}-m_{Z}^{2}}{\sin ^{2} \beta}+m_{Z}^{2}} .
$$

The general form of the one-loop stop/top contribution to the $\Delta \mathcal{M}_{22}^{2}$ element of the CPeven Higgs mass matrix, $\epsilon_{M S S M}$, is the same as in the $N=1 \mathrm{MSSM}$, see (2.17), and one can apply the same arguments about the relative unimportance of other MSSM loop contributions.

However, in the $N=2$ Higgs sector, there are additional loop contributions to the CP-even mass matrix from singlet and triplet adjoint scalars. We use the estimate of their contribution from $[38,49]$, where more details about the assumptions behind this estimate can be found:

$$
\begin{aligned}
\frac{32 \pi^{2}}{v^{2}} \Delta \epsilon_{N 2}= & \frac{g_{1}^{2}}{2} \ln \frac{m_{S}^{2}}{v^{2}}+\frac{3 g_{2}^{4}}{2} \ln \frac{m_{T}^{2}}{v^{2}} \\
& +\frac{g_{1}^{2} g_{2}^{2}}{m_{S}^{2}-m_{T}^{2}}\left[m_{S}^{2} \ln \frac{m_{S}^{2}}{v^{2}}-m_{T}^{2} \ln \frac{m_{T}^{2}}{v^{2}}-\left(m_{S}^{2}-m_{T}^{2}\right)\right] \\
& \stackrel{m_{S}^{2} \rightarrow m_{T}^{2}}{\longrightarrow} \frac{1}{2}\left(g_{1}^{4}+2 g_{1}^{2} g_{2}^{2}+3 g_{2}^{4}\right) \ln \frac{m_{T}^{2}}{v^{2}}
\end{aligned}
$$

where $m_{S}, m_{T}$ are the masses of the adjoint singlet and triplet scalars, respectively. In the last line of (2.24) we show the limiting value when these additional scalars are degenerate in mass. In our approximation, the total radiative correction to the mass matrix is then $\epsilon_{N 2}=\epsilon_{M S S M}+\Delta \epsilon_{N 2}$. The relative orders of magnitude of these two pieces can be estimated from their ratio when the adjoint singlet and triplet are mass degenerate:

$$
\frac{\epsilon_{M S S M}}{\Delta \epsilon_{N 2}} \simeq 36 \frac{\ln \left(\frac{M_{S}^{2}}{m_{t}^{2}}\right)}{\ln \left(\frac{m_{T}^{2}}{v^{2}}\right)} .
$$

This shows that $\Delta \tilde{\epsilon}_{N 2}$ is relatively unimportant for our current purposes: in our subsequent numerical estimates we use $m_{S}=m_{T}=1 \mathrm{TeV}$ as a default and we have neglected the scale dependance of the couplings between the Higgs fields and the singlet/triplet adjoint scalars [50].

Figure 2 displays the differences between the $h$ MSSM scenario in the $N=1$ case and the $h 2 \mathrm{MSSM}$ scenario in the $N=2$ case. The left panel of figure 2 compares the values of the mass of the heavier CP-even Higgs boson $H$ in the $h 2 \mathrm{MSSM}$ (red curve) and the 

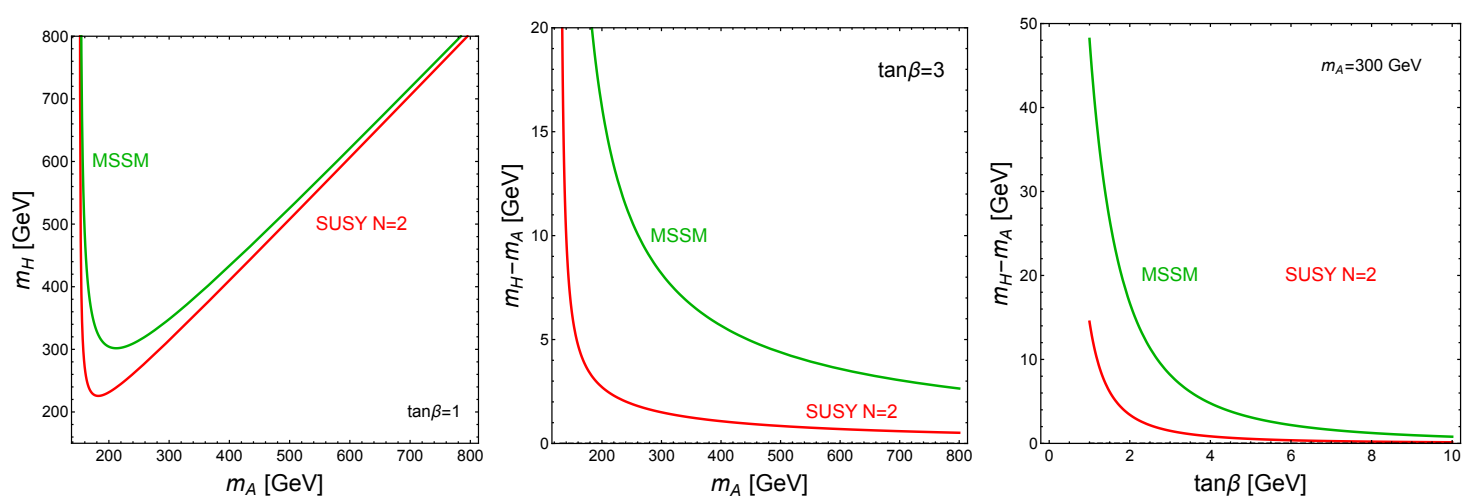

Figure 2. Left panel: the values of the mass of the heavier scalar Higgs boson $H$ as functions of $m_{A}$ for $\tan \beta=1$, when the leading one-loop radiative correction to the Higgs mass matrix, $\epsilon$, is chosen such that the lighter scalar Higgs boson $h$ has a mass of $125 \mathrm{GeV}$. Here and in the other panels, the red curve is for the $N=2 h 2 \mathrm{MSSM}$ scenario, and the green curve is for the $N=1 \mathrm{hMSSM}$. Middle panel: the mass differences $m_{H}-m_{A}$ for $m_{h}=125 \mathrm{GeV}$ in the $N=2 h 2 \mathrm{MSSM}$ scenario and in the $N=1 \mathrm{hMSSM}$ scenario as functions of $m_{A}$ for $\tan \beta=3$. Right panel: analogous curves as functions of $\tan \beta$ for $m_{A}=300 \mathrm{GeV}$.

$h \mathrm{MSSM}$ (green curve) as functions of $m_{A}$ for $\tan \beta=1$. We see that the $H$ boson has quite a different mass in the $h 2 \mathrm{MSSM}$ as compared to the $h \mathrm{MSSM}$. An interesting point is that, in both scenarios, $m_{H}$ diverges for some specific value of $m_{A}$ slightly above $125 \mathrm{GeV}$, the exact value depending on $\tan \beta$ as shown in (2.23). This corresponds to the fact that there is no value of $\epsilon$ that satisfies the requirement $m_{h}=125 \mathrm{GeV}$ for a region of the $\left(m_{A}, \tan \beta\right)$ parameter plane. However, in the $N=2 h 2 \mathrm{MSSM}$ scenario, the divergence in the required value of $m_{H}$ is less severe.

The eagle-eyed reader will notice that the red curve for $m_{H}$ in the left panel of figure 2 lies extremely close to the green curve for $m_{A}$. As we see in the other panels of figure 2 , it is a general feature of the $h 2 \mathrm{MSSM}$ that $m_{H}-m_{A}$ is smaller than in the MSSM. In the middle panel of figure 2, we plot the mass splitting $m_{H}-m_{A}$ in the $h 2 \mathrm{MSSM}$ as a function of $m_{A}$ for $\tan \beta=3$ (red curve). The right panel of figure 2 shows the corresponding calculation of the mass splitting $m_{H}-m_{A}$ in the $h 2 \mathrm{MSSM}$ as a function of $\tan \beta$ for $m_{A}=300 \mathrm{GeV}$ (red curve). The similar feature of a smaller magnitude is again apparent. The fact that $m_{H}-m_{A}$ is small is relevant to the LHC experimental searches for $H / A \rightarrow \tau^{+} \tau^{-}$that we discuss later, since they assume that this mass difference is smaller than their experimental resolution.

Figure 3 displays contours of $\cos ^{2}(\beta-\alpha)$ in the $\left(m_{A}, \tan \beta\right)$ plane for the $h$ MSSM scenario (left panel) and the $N=2 h 2 \mathrm{MSSM}$ scenario (right panel). This quantity determines the coupling of the heavier CP-even Higgs boson $H$ to the electroweak gauge sector. We can see that this coupling is significantly reduced in the h2MSSM, compared to the $h \mathrm{MSSM}$, reducing the impact of the experimental constraints, as we also discuss later. 

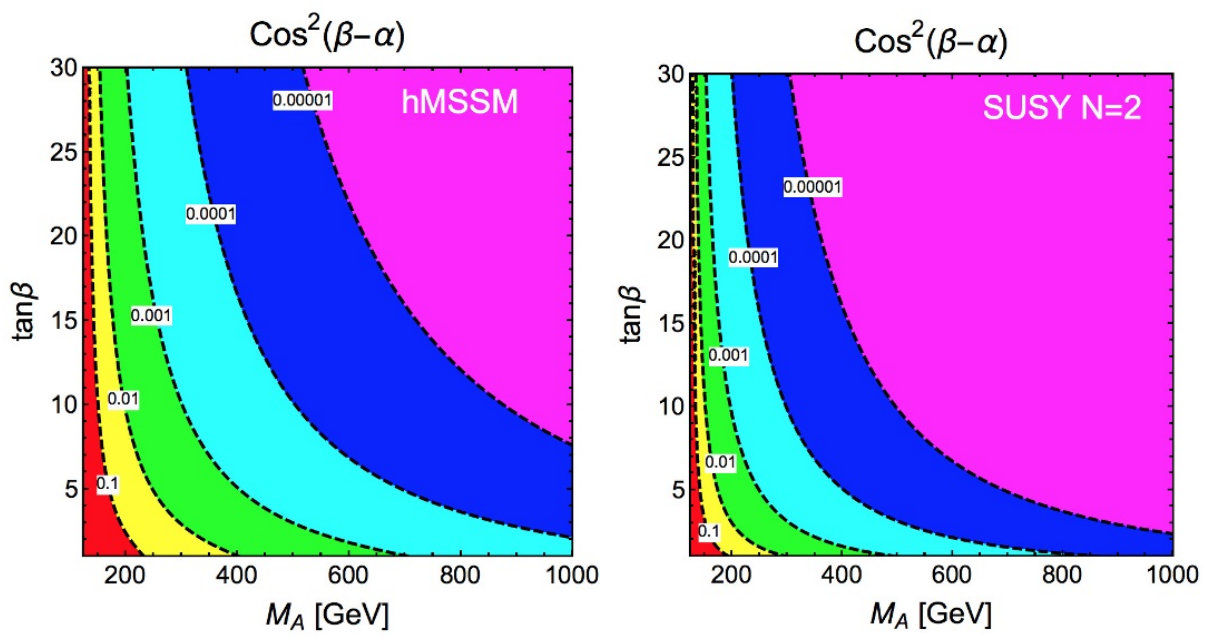

Figure 3. Contours of $\cos ^{2}(\beta-\alpha)$ in the $\left(m_{A}, \tan \beta\right)$ plane for the $h$ MSSM scenario (left panel) and the $N=2 h 2 \mathrm{MSSM}$ scenario (right panel).

\subsection{The stop sector in the $h$ MSSM and the $h 2 \mathrm{MSSM}$}

Thus far, we have simply assumed that the stop sector is such that $m_{h}=125 \mathrm{GeV}$. Now we study what properties the stop sector must have in order for this to be possible. We recall from (2.17) that the two relevant parameters in $\epsilon_{M S S M}$ are $M_{S U S Y}$ and $X_{t}$. As can be seen there, the radiative correction increases monotonically with $M_{S U S Y}$, but depends in a nontrivial and nonlinear way on $X_{t}$. This means that any statement about the required size of $M_{S U S Y}$ is dependent on the assumed value of $X_{t}$, and more than one value of $X_{t}$ may yield $m_{h}=125 \mathrm{GeV}$ with the same value of $M_{S U S Y}$. These remarks apply to both the $h \mathrm{MSSM}$ and the $h 2 \mathrm{MSSM}$. Looking at figure 1 , however, we recall that the tree-level value of $m_{h}$ is larger in the $N=2$ extension of the MSSM than in its $N=1$ version. This implies that the required magnitude of $\epsilon_{M S S M}$ is smaller in the $h 2 \mathrm{MSSM}$ than in the $h \mathrm{MSSM}$ and hence that, for any fixed value of $X_{t}$, the required value of $M_{S U S Y}$ is also smaller, as we now discuss in more detail.

We display in figure 4 the values of $M_{S U S Y}$ that are required in the $h \mathrm{MSSM}$ (green dotted lines) and the $h 2 \mathrm{MSSM}$ (red full lines) to yield $m_{h}=125 \mathrm{GeV}$, as functions of $X_{t} / M_{S U S Y}$. The first point visible in these plots is that the required value of $M_{S U S Y}$ is very sensitive to $X_{t}$, in both scenarios. It is occasionally said that $m_{h}=125 \mathrm{GeV}$ requires, within the MSSM, values of $M_{S U S Y}$ in the multi-TeV range. We see that this is true in the $h$ MSSM for $X_{t}=0$ and $\tan \beta=1$ (left panel), but is not true in general. For example, as seen in the middle panel, for most values of $X_{t}, M_{S U S Y}<1000 \mathrm{GeV}$ is sufficient in the $h \mathrm{MSSM}$ if $\tan \beta=3$, and even $M_{S U S Y}<600 \mathrm{GeV}$ for a suitable choice of $X_{t}$. The trend to lower $M_{S U S Y}$ continues for $\tan \beta=10$ (right panel) and larger.

However, the key new point of our analysis is that the required values of $M_{S U S Y}$ are indeed significantly lower in the $h 2 \mathrm{MSSM}$ than in the $h \mathrm{MSSM}$. For example, $M_{S U S Y}=$ $1000 \mathrm{GeV}$ is now possible for $\tan \beta=1$ (left panel), $M_{S U S Y}=200 \mathrm{GeV}$ is possible for $\tan \beta=3$ (middle panel), and even smaller values of $M_{S U S Y}$ are possible for $\tan \beta=10$ (right panel). 

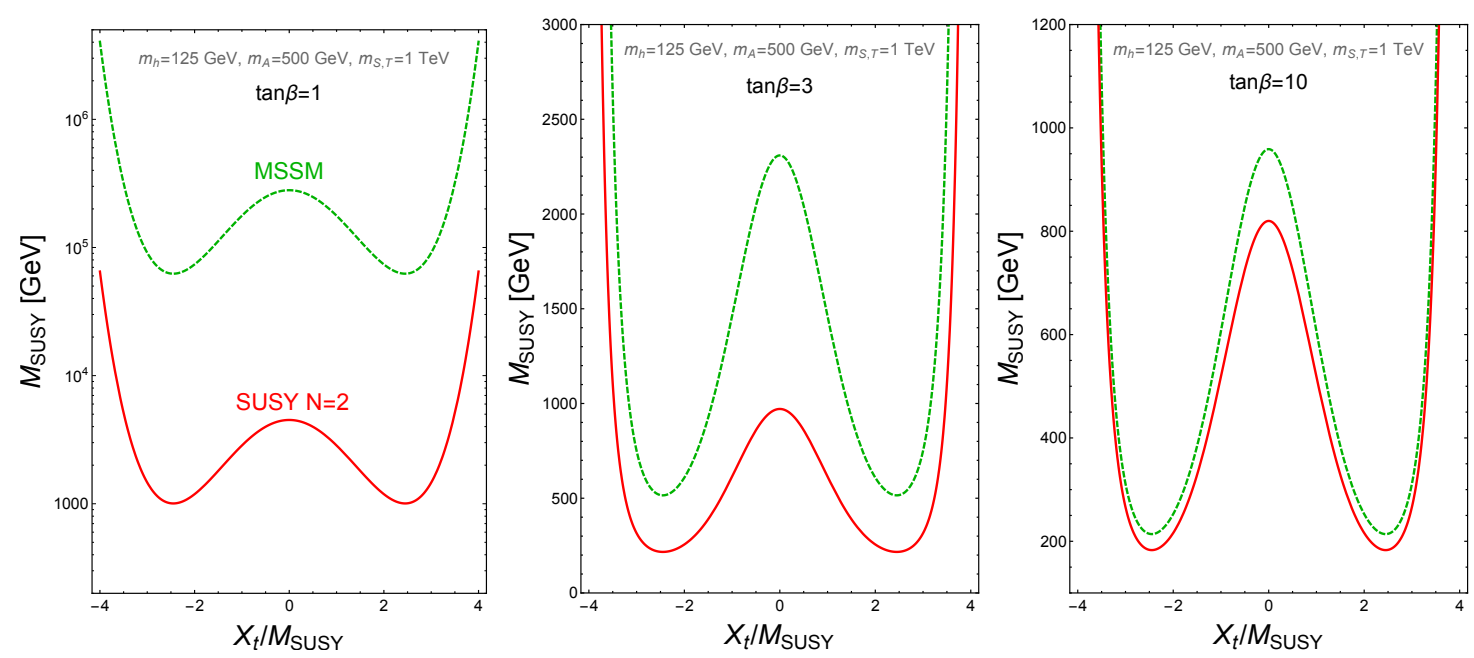

Figure 4. Contours of $M_{S U S Y}$ as functions of $X_{t} / M_{S U S Y}$ that yield $m_{h}=125 \mathrm{GeV}$ in the $h \mathrm{MSSM}$ scenario (green dotted lines) and the $N=2 h 2 \mathrm{MSSM}$ scenario (red full lines). The left panel is for $m_{A}=500 \mathrm{GeV}$ and $\tan \beta=1$, the middle panel is for $m_{A}=500 \mathrm{GeV}$ and $\tan \beta=3$, and the right panel is for $m_{A}=500 \mathrm{GeV}$ and $\tan \beta=10$, and we assume $m_{S}=m_{T}=1 \mathrm{TeV}$ in the $h 2 \mathrm{MSSM}$ cases.

Some caveats are in order. As discussed earlier, in this analysis we consider only the stop contributions to the $\Delta \mathcal{M}_{22}^{2}$ element in the CP-even Higgs mass matrix. However, as argued previously, the contributions to other entries in this mass matrix are subdominant, at least for small $\mu$. Secondly, we have neglected two- and multi-loop effects, but these should not change our qualitative results. Finally, as also argued previously, the specifically $N=2$ one-loop corrections due to the adjoint scalar fields are also expected not to affect significantly our results: for definiteness, we have chosen $m_{S}=m_{T}=1 \mathrm{TeV}$ in the $h 2 \mathrm{MSSM}$ plots in the right panels of figure 4.

A different way of visualizing our results for the $h$ MSSM and h2MSSM is shown in figure 5. Comparing the two panels, we see that much lower values of $M_{S U S Y}$ are required for the maximal-mixing scenario $X_{t}=\sqrt{6} M_{S U S Y}$ (right panel) than for $X_{t}=0$ (left panel). However, the most striking and novel feature is that, as remarked above, the $h 2 \mathrm{MSSM}$ requires much smaller values of $M_{S U S Y}$. When $X_{t}=0$ (left panel), for $\tan \beta \sim 3$ in the $h \mathrm{MSSM}$ values of $M_{S U S Y} \sim 2000 \mathrm{GeV}$ are required, whereas $M_{S U S Y}>1000 \mathrm{GeV}$ are sufficient in the $h 2 \mathrm{MSSM}$. In the maximal-mixing scenario these values are reduced to $M_{S U S Y} \sim 900 \mathrm{GeV}$ in the $h \mathrm{MSSM}$ and $M_{S U S Y} \sim 250 \mathrm{GeV}$ in the $h 2 \mathrm{MSSM}$.

\section{Constraints from LHC measurements}

In light of these differences between the masses and couplings of the Higgs bosons in the $h 2 \mathrm{MSSM}$ and $h \mathrm{MSSM}$, we now examine the impacts of LHC constraints in the $\left(m_{A}, \tan \beta\right)$ plane. 

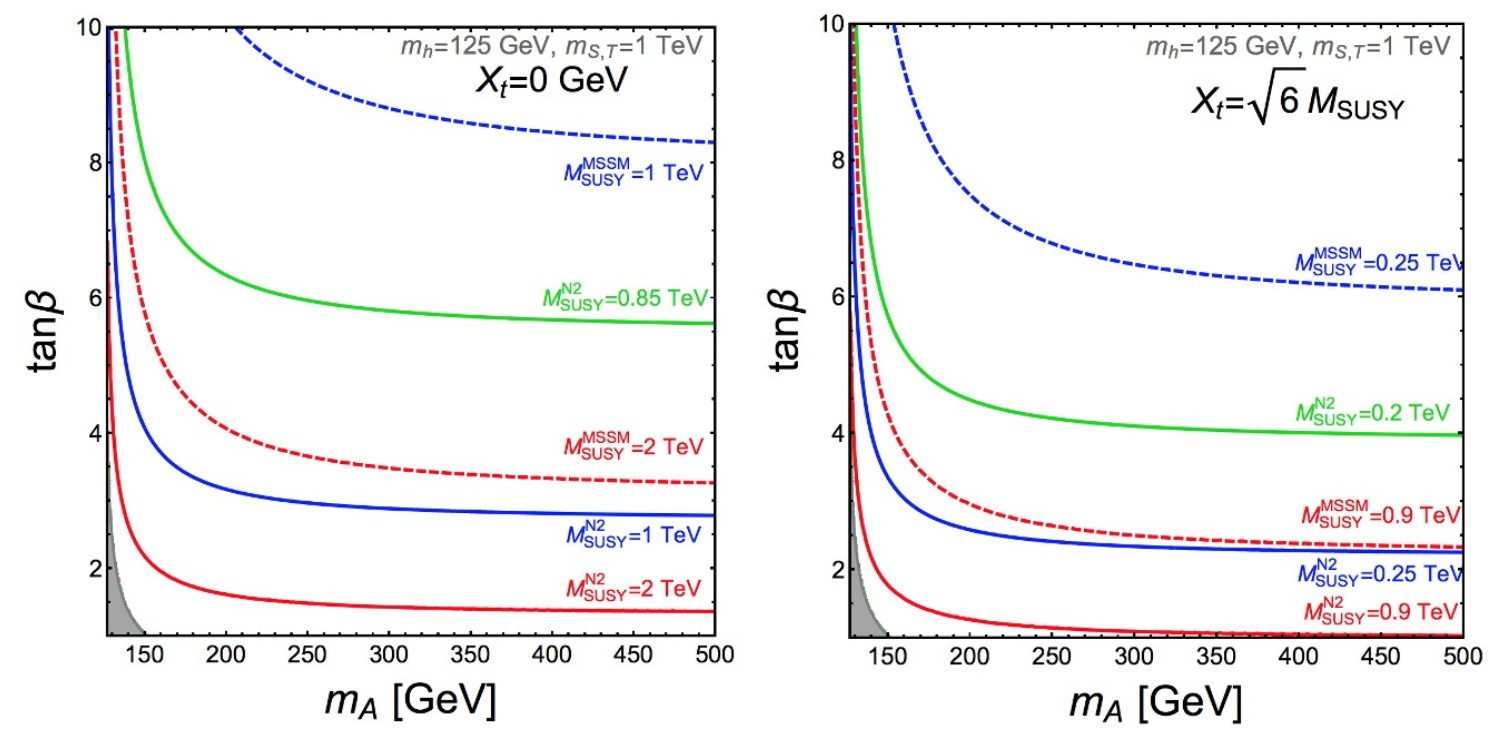

Figure 5. Contours of $M_{S U S Y}$ as functions of $m_{A}$ and $\tan \beta$ that yield $m_{h}=125 \mathrm{GeV}$ in the $h$ MSSM scenario (dotted lines) and the $N=2 h 2 \mathrm{MSSM}$ scenario (full lines), assuming $m_{S}=m_{T}=$ $1 \mathrm{TeV}$ in the $h 2 \mathrm{MSSM}$ cases. The left panel is for $X_{t}=0$, and the right panel is for the maximalmixing scenario with $X_{t}=\sqrt{6} M_{S U S Y}$. The grey areas correspond to the region disallowed in our scenarios, cf, (2.23).

\subsection{Constraints from $H / A / H^{ \pm}$searches}

Since the mixing angle of the tree-level scalar mass matrix is exactly $\alpha=\beta-\pi / 2$ in the $h 2 \mathrm{MSSM}$, the heavy Higgs bosons decouple from pairs of gauge bosons at this level, and the loop-induced $H W^{+} W^{-}, H Z^{0} Z^{0}$ and $A Z h$ couplings are relatively small. The limits in the $\left(m_{A}, \tan \beta\right)$ plane of the $N=1 \mathrm{hMSSM}$ coming from $H$ decays to $W^{+} W^{-}$and $Z^{0} Z^{0}$ and $A$ decay to $Z h[42-45,51]$ are therefore not applicable to the $h 2 \mathrm{MSSM}$. Only the constraints from $H, A$ and $H^{ \pm}$couplings to Standard Model fermions are applicable to the h2MSSM. As we have seen, the $H-A$ mass difference is smaller in the h2MSSM than in the $h \mathrm{MSSM}$, so the LHC constraints on $A / H \rightarrow \tau^{+} \tau^{-}$are applicable without modification. This is shown in figure 6 as a grey excluded region excluding a range of $m_{A}$ for $\tan \beta \gtrsim 7$. We do not display the constraint from $H^{ \pm} \rightarrow \tau^{ \pm} \nu$ searches, which exclude a small region at small $m_{A}$ and large $\tan \beta$ that is contained within the grey area [42-45].

\subsection{Constraints from $h(125)$ coupling measurements}

The couplings of the Standard Model-like Higgs boson $h(125)$ [37] can be analysed using the following effective field theory (EFT):

$$
\begin{aligned}
\mathcal{L}_{\mathrm{h}-\mathrm{EFT}}= & \kappa_{V} g_{h W W} h W_{\mu}^{+} W^{-\mu}+\kappa_{V} g_{h Z Z} h Z_{\mu}^{0} Z^{0 \mu} \\
& -\kappa_{t} y_{t} h \bar{t}_{L} t_{R}-\kappa_{t} y_{c} h \bar{c}_{L} c_{R}-\kappa_{b} y_{b} h \bar{b}_{L} b_{R}-\kappa_{b} y_{\tau} h \bar{\tau}_{L} \tau_{R}+\text { h.c. },
\end{aligned}
$$

where $y_{t, c, b, \tau}=m_{t, c, b, \tau} / v$ are the Standard Model Yukawa couplings in the mass eigenbasis, the subscripts $L / R$ label the left and right chirality states of the fermions, and we consider 


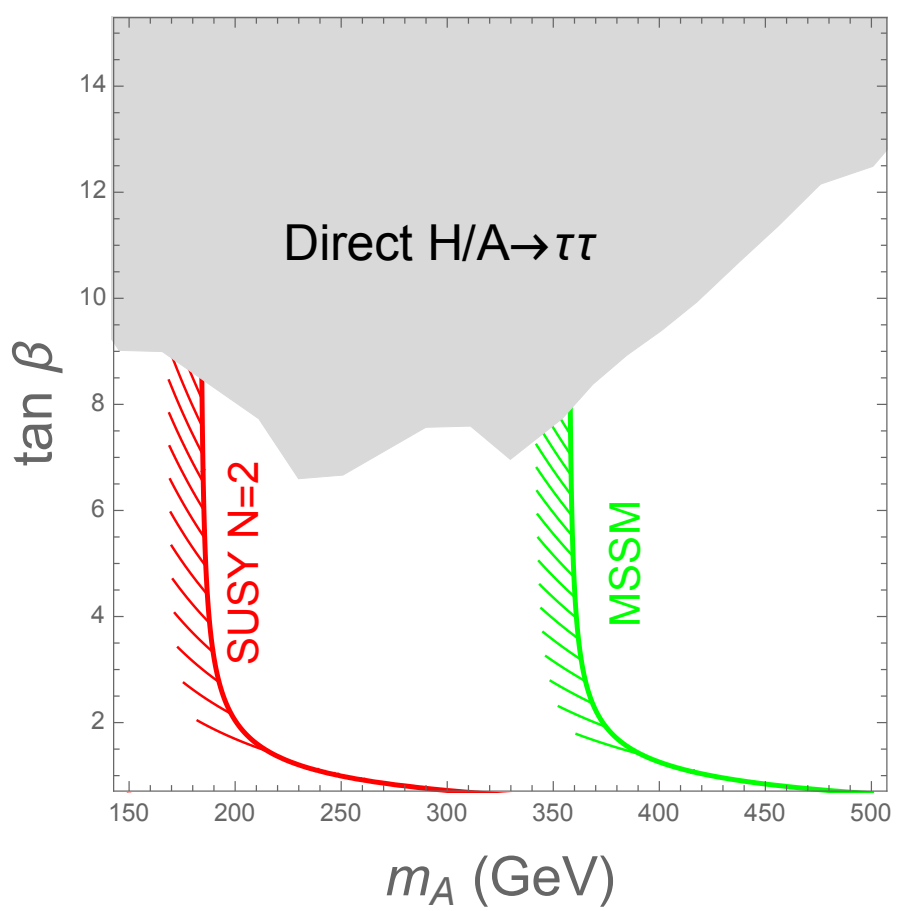

Figure 6. We show in grey the direct exclusion from searches for heavy scalars in the $H / A \rightarrow \tau \tau$ final state, which apply to both the $h \mathrm{MSSM}$ and the $h 2 \mathrm{MSSM}$. We also show the indirect bounds from measurements of Higgs couplings to fermions and massive bosons at Run 1 of the LHC for the $h \mathrm{MSSM}$ (green) and $N=2 h 2 \mathrm{MSSM}$ (red), where the regions to the left of the lines are excluded in each case.

only the fermions with the largest couplings to the Higgs boson. The quantities $g_{h W W}=$ $2 m_{W}^{2} / v$ and $g_{h Z Z}=m_{Z}^{2} / v$ are the couplings of $h$ to the electroweak gauge bosons, and $v$ is the vacuum expectation value of the Higgs field. The parameters $\kappa_{X}$ are the free parameters of this EFT.

These parameters can be constrained using the Higgs signal strengths in various channels, denoted by $X X$ :

$$
\mu_{X} \equiv \frac{\sigma(p p \rightarrow h) \times \mathrm{BR}(h \rightarrow X X)}{\sigma(p p \rightarrow h)_{\mathrm{SM}} \times \mathrm{BR}(h \rightarrow X X)_{\mathrm{SM}}},
$$

as measured in all the Higgs production/decay channels available from the LHC Run 1. A full analysis requires performing an appropriate three-parameter fit in the threedimensional $\left(\kappa_{V}, \kappa_{t}, \kappa_{b}\right)$ space, where we assume that $\kappa_{c}=\kappa_{t}, \kappa_{\tau}=\kappa_{b}$, which is consistent with the current experimental accuracies, and $\kappa_{V}=\kappa_{W}=\kappa_{Z}$, the custodial symmetry relations that should hold to a good approximation in the supersymmetric models of interest.

In our two supersymmetric models, the $N=1 \mathrm{MSSM}$ and the $N=2 \mathrm{~h} 2 \mathrm{MSSM}$ scenario, the $\kappa$ parameters take the following similar forms:

$$
\kappa_{V}=\sin (\beta-\alpha), \quad \kappa_{t}=\frac{\cos \alpha}{\sin \beta}, \quad \kappa_{b}=-\frac{\sin \alpha}{\cos \beta}
$$


where $\alpha$ is the rotation angle that diagonalizes the Higgs mass-squared matrix in the $h \mathrm{MSSM}$ or $h 2 \mathrm{MSSM}$, respectively, after including the dominant one-loop radiative corrections as discussed above. The expressions (3.3) do not include the effects of subdominant loop corrections, which may not be negligible if the supersymmetric particles are not very heavy, in which case there are direct radiative corrections to the Higgs couplings that are not contained in the expression of the mass matrix. We neglect such possible effects in the present study.

At tree level, $\alpha$ only depends on two unknown quantities, namely $\tan \beta$ and $m_{A}$. Moreover, only two of the three quantities $\kappa_{V}, \kappa_{t}$ and $\kappa_{b}$ are independent. This is still the case when we include the dominant one-loop radiative corrections and fix $m_{h}=125 \mathrm{GeV}$ as discussed above. In both the $h \mathrm{MSSM}$ and the $h 2 \mathrm{MSSM}$ we can derive $\kappa_{V}\left(\tan \beta, m_{A}\right)$, $\kappa_{t}\left(\tan \beta, m_{A}\right)$ and $\kappa_{b}\left(\tan \beta, m_{A}\right)$ for any pair of values of $\left(\tan \beta, m_{A}\right)$.

The values may be derived by plugging the explicit expressions for $\alpha_{M S S M}$ in (2.19) and $\alpha_{N 2}$ in (2.22) into (3.3). Alternatively, one can proceed directly from the MSSM or $N=2$ mass-squared matrix, associating the mass eigenvalue $m_{h}$ with the normalized eigenvector $V_{h}=\left(V_{h 1}, V_{h 2}\right)$ such that the physical field is $h=V_{h i} H_{i}$ with $i=1,2$ and the mass eigenvalue $m_{H}$ with the normalized eigenvector $V_{H}=\left(V_{H 1}, V_{H 2}\right)$ such that the physical field is $H=V_{H i} H_{i}$ with $i=1,2$. We then have

$$
\begin{aligned}
\kappa_{t} & =\frac{1}{\sin \beta} V_{h 2}\left(\tan \beta, m_{A}\right), \quad \kappa_{b}=\frac{1}{\cos \beta} V_{h 1}\left(\tan \beta, m_{A}\right), \\
\kappa_{V} & =\sin \beta V_{h 2}\left(\tan \beta, m_{A}\right)+\cos \beta V_{h 1}\left(\tan \beta, m_{A}\right) .
\end{aligned}
$$

In terms of $\tan \beta$ we find

$$
\begin{aligned}
\kappa_{t} & =\frac{\sqrt{1+\tan ^{2} \beta}}{\tan \beta} V_{h 2}\left(\tan \beta, m_{A}\right), \quad \kappa_{b}=\sqrt{1+\tan ^{2} \beta} V_{h 1}\left(\tan \beta, m_{A}\right), \\
\kappa_{V} & =\frac{1}{\sqrt{1+\tan ^{2} \beta}}\left(\tan \beta V_{h 2}\left(\tan \beta, m_{A}\right)+V_{h 1}\left(\tan \beta, m_{A}\right)\right),
\end{aligned}
$$

where in the case of the $h \mathrm{MSSM}$ :

$$
\begin{aligned}
V_{h 2}^{M S S M}\left(\tan \beta, m_{A}\right) & =\frac{1}{\sqrt{1+\left(\frac{\left(m_{A}^{2}+m_{Z}^{2}\right) \tan \beta}{m_{Z}^{2}-m_{h}^{2}\left(1+\tan ^{2} \beta\right)+m_{A}^{2} \tan ^{2} \beta}\right)^{2}}}, \\
V_{h 1}^{M S S M}\left(\tan \beta, m_{A}\right) & =\frac{\left(m_{A}^{2}+m_{Z}^{2}\right) \tan \beta}{m_{Z}^{2}-m_{h}^{2}\left(1+\tan ^{2} \beta\right)+m_{A}^{2} \tan ^{2} \beta} V_{h 2},
\end{aligned}
$$

and in the case of the $N=2$ h2MSSM:

$$
\begin{aligned}
V_{h 2}^{N 2}\left(\tan \beta, m_{A}\right) & =\frac{1}{\sqrt{1+\left(\frac{\left(m_{A}^{2}-m_{Z}^{2}\right) \sin 2 \beta}{m_{A}^{2}-2 m_{h}^{2}+m_{Z}^{2}+\left(m_{Z}^{2}-m_{A}^{2}\right) \cos 2 \beta}\right)^{2}}}, \\
V_{h 1}^{N 2}\left(\tan \beta, m_{A}\right) & =\frac{\left(m_{A}^{2}-m_{Z}^{2}\right) \sin 2 \beta}{m_{A}^{2}-2 m_{A}^{2}+m_{Z}^{2}+\left(m_{Z}^{2}-m_{A}^{2}\right) \cos 2 \beta} V_{h 2} .
\end{aligned}
$$

These results can be used to apply the constraints on Higgs couplings derived from a combination of CMS and ATLAS data at Run1 [52]. In particular, the analysis relevant to constraining the $h \mathrm{MSSM}$ and $h 2 \mathrm{MSSM}$ scenarios tests for deviations from the Standard Model 
in couplings to up- and down-type quarks and to vector bosons via the ratios $\lambda_{d u}$ and $\lambda_{V u}$ :

$$
\begin{aligned}
\lambda_{d u} & =\frac{\kappa_{d}}{\kappa_{u}}=0.92_{-0.12}^{+0.12}, \\
\lambda_{V u} & =\frac{\kappa_{V}}{\kappa_{u}}=1.00_{-0.12}^{+0.13} .
\end{aligned}
$$

The results of this fit are shown in figure 6 , where the excluded region in the $h$ MSSM lies to the left of the green line, whereas in the $N=2$ case the bounds (in red) are very much weakened.

We conclude from figure 6 that $m_{A} \gtrsim 200 \mathrm{GeV}$ is allowed in the $h 2 \mathrm{MSSM}$ for $\tan \beta \in$ $(2,8)$, whereas $m_{A} \gtrsim 350 \mathrm{GeV}$ would be required in the $h \mathrm{MSSM}$.

\subsection{Constraints from $\Gamma(h \rightarrow g g, \gamma \gamma)$}

We now analyze the corrections to the couplings of the SM-like Higgs boson to gluons and photons that arise at the loop level, and the corresponding constraints on the $h \mathrm{MSSM}$ and h2MSSM.

The decay width of the Standard Model-like $h(125)$ into pairs of gluons and photons can be expressed as $[53,54]$ :

$$
\Gamma(h \rightarrow g g)=\frac{G_{F} \alpha_{s}^{2} m_{h}^{3}}{64 \sqrt{2} \pi^{3}}\left|\sum_{i} A_{i}^{g g}\left(\tau_{i}\right)\right|^{2}, \Gamma(h \rightarrow \gamma \gamma)=\frac{G_{F} \alpha^{2} m_{h}^{3}}{128 \sqrt{2} \pi^{3}}\left|\sum_{i} A_{i}^{\gamma \gamma}\left(\tau_{i}\right)\right|^{2},
$$

where the variable $\tau_{i} \equiv m_{h}^{2} / 4 m_{i}^{2}, m_{i}$ being the mass of the particle propagating in the loop. In the case of the loops for the $h g g$ coupling, whereas one has only contributions from quarks in the Standard Model, in the MSSM additional contributions are provided by the scalar partners of those quarks. The normalized amplitudes of these two contributions are

$$
A_{f}^{g g}=g_{h f f} F_{1 / 2}\left(\tau_{f}\right) \quad, A_{\tilde{f}_{i}}^{g g}=g_{h \tilde{f}_{i} \tilde{f}_{i}} \frac{M_{Z}^{2}}{m_{\tilde{f}_{i}}^{2}} F_{0}\left(\tau_{\tilde{f}_{i}}\right) .
$$

In the case of the loop for the $h \gamma \gamma$ coupling, in the Standard Model the $W$ boson and charged fermions are the only contributors, whereas in the MSSM there are additional contributions from the two chargino fermionic fields, the scalar partners of the fermions and the charged Higgs boson. The normalized amplitudes of these contributions are

$$
\begin{aligned}
& A_{W}^{\gamma \gamma}=g_{\Phi W W} F_{1}\left(\tau_{W}\right), \quad A_{f}^{\gamma \gamma}=N_{c} Q_{f}^{2} g_{\Phi f f} F_{1 / 2}\left(\tau_{f}\right), \quad A_{\chi_{i}}^{\gamma \gamma}=g_{\Phi \chi_{i}^{+} \chi_{i}^{-}} \frac{M_{W}}{m_{\chi_{i}}} F_{1 / 2}\left(\tau_{\chi_{i}}\right), \\
& A_{\tilde{f}_{i}}^{\gamma \gamma}=N_{c} Q_{f}^{2} g_{\Phi \tilde{f}_{i} \tilde{f}_{i}} \frac{M_{Z}^{2}}{m_{\tilde{f}_{i}}^{2}} F_{0}\left(\tau_{\tilde{f}_{i}}\right), \quad A_{H^{ \pm}}^{\gamma \gamma}=g_{\Phi H^{+} H^{-}} \frac{M_{W}^{2}}{M_{H^{ \pm}}^{2}} F_{0}\left(\tau_{H^{ \pm}}\right)
\end{aligned}
$$

where $N_{c}$ is the color factor and $Q_{f}$ the electric charge of the fermion or sfermion in units of the proton charge.

The spin 1, 1/2 and 0 amplitudes are [53]

$$
\begin{aligned}
F_{1}(\tau) & =\left[2 \tau^{2}+3 \tau+3(2 \tau-1) f(\tau)\right] / \tau^{2}, \\
F_{1 / 2}(\tau) & =-2[\tau+(\tau-1) f(\tau)] / \tau^{2}, \\
F_{0}(\tau) & =[\tau-f(\tau)] / \tau^{2}
\end{aligned}
$$


with the function $f(\tau)$ defined as

$$
f(\tau)= \begin{cases}\arcsin ^{2} \sqrt{\tau} & \tau \leq 1 \\ -\frac{1}{4}\left[\log \frac{1+\sqrt{1-\tau^{-1}}}{1-\sqrt{1-\tau^{-1}}}-i \pi\right]^{2} & \tau>1 .\end{cases}
$$

The amplitudes are real when $m_{h}<2 m_{i}$, but are complex above that threshold. In the regime $\tau \ll 1$, i.e., heavy masses in the loop, the amplitudes reach asymptotic values

$$
F_{1} \rightarrow+7, \quad F_{1 / 2} \rightarrow-\frac{4}{3} \text { and } F_{0} \rightarrow-\frac{1}{3} .
$$

Standard Model particle loops give finite contributions in the heavy-mass limit, whereas the new supersymmetric contributions decouple in the limit of large mass, since their amplitudes $A_{i}$ are divided by their masses.

As we have discussed in the previous section, the top quark superpartners are responsible for a substantial shift in the tree-level Higgs mass of $\sim 34 \mathrm{GeV}$ in the h2MSSM (and more in the $h \mathrm{MSSM}$ ). We will focus in the following on the loop-level correction to the $h g g$ and $h \gamma \gamma$ couplings due to the stops, neglecting other potential supersymmetric contributions.

The loop-level corrections from stops to Higgs production via gluon-gluon fusion and to $h \rightarrow \gamma \gamma$ decay are given, respectively, by

$$
\frac{\sigma(g g \rightarrow h)}{\sigma^{S M}(g g \rightarrow h)} \simeq \frac{\Gamma(h \rightarrow g g)}{\Gamma^{S M}(h \rightarrow g g)} \simeq\left|\kappa_{g}\right|^{2}, \quad \frac{\Gamma(h \rightarrow \gamma \gamma)}{\Gamma^{S M}(h \rightarrow \gamma \gamma)} \simeq\left|\kappa_{\gamma}\right|^{2},
$$

with

$$
\kappa_{g}=1+\frac{A_{\tilde{t}_{1}}^{g g}+A_{\tilde{t}_{2}}^{g g}}{\sum_{i \in S M} A_{i}^{g g}}, \quad \kappa_{\gamma}=1+\frac{A_{\tilde{t}_{1}}^{\gamma \gamma}+A_{\tilde{t}_{2}}^{\gamma \gamma}}{\sum_{i \in S M} A_{i}^{\gamma \gamma}} .
$$

It has been shown that, to a good approximation [55], $\kappa_{g, \gamma}$ reduce to

$$
\kappa_{g} \simeq 1+\frac{A_{\tilde{t}}}{\sum_{i \in S M} A_{i}^{g g}}, \quad \kappa_{\gamma} \simeq 1+\frac{N_{c} Q_{\tilde{t}}^{2} A_{\tilde{t}}}{\sum_{i \in S M} A_{i}^{\gamma \gamma}},
$$

where

$$
A_{\tilde{t}}=-\frac{1}{3}\left(\frac{m_{t}^{2}}{m_{\tilde{t}_{1}}^{2}}+\frac{m_{t}^{2}}{m_{\tilde{t}_{2}}^{2}}-\frac{1}{4} \sin ^{2}\left(2 \theta_{t}\right) \frac{\left(m_{\tilde{t}_{2}}^{2}-m_{\tilde{t}_{1}}^{2}\right)^{2}}{m_{\tilde{t}_{1}}^{2} m_{\tilde{t}_{2}}^{2}}\right),
$$

with $\theta_{t}$ the mixing angle of the scalar mass matrix. We remind the reader that the physical stop masses are

$$
m_{\tilde{t}_{1}, \tilde{t}_{2}}^{2}=m_{t}^{2}+\frac{1}{2}\left[m_{\tilde{t}_{L}}^{2}+m_{\tilde{t}_{R}}^{2} \mp \sqrt{\left(m_{\tilde{t}_{L}}^{2}-m_{\tilde{t}_{R}}^{2}\right)^{2}+\left(2 m_{t} X_{t}\right)^{2}}\right],
$$

where $X_{t}=A_{t}-\mu / \tan \beta$, and $A_{t}, m_{\tilde{t}_{R}}$ and $m_{\tilde{t}_{L}}$ are parameters of the soft supersymmetrybreaking Lagrangian, and the squark mixing angle, $\theta_{t}$, is defined by

$$
\sin 2 \theta_{t}=\frac{2 m_{t} X_{t}}{m_{\tilde{t}_{1}}^{2}-m_{\tilde{t}_{2}}^{2}}, \quad \cos 2 \theta_{t}=\frac{m_{\tilde{t}_{L}}^{2}-m_{\tilde{t}_{R}}^{2}}{m_{\tilde{t}_{1}}^{2}-m_{\tilde{t}_{2}}^{2}} .
$$


The stop sector can be parametrised by the three inputs $m_{\tilde{t}_{L}}, m_{\tilde{t}_{R}}$ and $X_{t}$ or, alternatively, by the physical stop masses $m_{\tilde{t}_{1}}, m_{\tilde{t}_{2}}$ and $X_{t}$. If the mixing parameter is large, the two stop masses are strongly split, $m_{\tilde{t}_{1}} \ll m_{\tilde{t}_{2}}$, and the $\tilde{t}_{1}$ has a large coupling to the $h(125)$ state, $g_{\tilde{t}_{1} \tilde{t}_{1}} \propto m_{t} X_{t}$.

If we consider the $\left[m_{\tilde{t}_{1}}, m_{\tilde{t}_{2}}\right]$ plane for fixed values of $m_{A}$ and $\tan \beta$, we can fix $X_{t}^{2}$ by the requirement that $m_{h}=125 \mathrm{GeV}$ when just the dominant stop contributions to the radiative corrections in the MSSM Higgs sector are considered [56]. In this case, the shift of the Higgs mass is given by (2.17) and (2.24) in the $h$ MSSM and $h 2 \mathrm{MSSM}$, respectively. There are at most two solutions for $X_{t}^{2}$, denoted by $\left|X_{t}^{\max }\right|$ and $\left|X_{t}^{\min }\right|$. Having traded the stop mixing parameter by the requirement $m_{h}=125 \mathrm{GeV}$, we can now compute the couplings between the stops and the $h(125)$ and then $\kappa_{g, \gamma}$.

The available experimental constraints on $\kappa_{\gamma}$ are shown in green (red) for the $h \mathrm{MSSM}$ ( $h 2 \mathrm{MSSM}$ ) in figure 7 for $m_{A}=500 \mathrm{GeV}$ and $\tan \beta=1.5$ (upper panels), $\tan \beta=5$ (middle panels) and $\tan \beta=10$ (lower panels). In the case of the h2MSSM, we always consider a generic common adjoint scalar mass $m_{S}=m_{T}=1 \mathrm{TeV}$. The constraints on $\kappa_{g}$ are less severe than those on $\kappa_{\gamma}$, so we do not display them in figure 7 .

The Higgs mass requirement has, in general, zero, one or two solutions for $X_{t}^{2}$, and it is possible that one or more of them might be in conflict with the constraint coming from the soft masses:

$$
\left(m_{\tilde{t}_{L}}^{2}-m_{\tilde{t}_{R}}^{2}\right)^{2}=\left(m_{\tilde{t}_{1}}^{2}-m_{\tilde{t}_{2}}^{2}\right)^{2}-\left(2 m_{t} X_{t}\right)^{2},
$$

from which we can derive the maximum allowed value for $X_{t},\left|X_{t}^{\text {soft }}\right|$, which is given by

$$
X_{t}^{\text {soft }, 2}=\frac{\left(m_{\tilde{t}_{1}}^{2}-m_{\tilde{t}_{2}}^{2}\right)^{2}}{4 m_{t}^{2}} .
$$

When scanning the $\left(m_{\tilde{t}_{1}}, m_{\tilde{t}_{2}}\right)$ plane, we must ensure that our solutions in $X_{t}$ are below this maximal value. The grey regions in figure 7 with dotted (full) border contours are forbidden by this consideration in the case of the $h$ MSSM ( $h 2 \mathrm{MSSM})$. There are no values of $X_{t}$ able to accommodate $m_{h}=125 \mathrm{GeV}$ in the hMSSM (h2MSSM) in the regions at low $m_{\tilde{t}_{1}}$ and/or $m_{\tilde{t}_{2}}$ that are shaded yellow (blue).

The left panels of figure 7 consider the maximal value of $X_{t}$ allowing $m_{h}=125 \mathrm{GeV}$, including the case where there is only one possible choice for $X_{t}$. The right panels of figure 7 consider the minimal value of $X_{t}$ allowing $m_{h}=125 \mathrm{GeV}$, including the case where there is only one possible choice for $X_{t}$. This explains the particular shape of the grey region for relatively high stop masses.

The current constraints on $\kappa_{g, \gamma}$ in the $h \mathrm{MSSM}$ and the $h 2 \mathrm{MSSM}$ are outlined in green (red) in figure 7. We see that they are generally weak. Indeed, for $m_{A}=500 \mathrm{GeV}$ and $\tan \beta=1.5$ (top two panels) there is no constraint at all. However, for higher values of $\tan \beta$ (middle and bottom panels) these constraints do exclude some scenarios with low supersymmetry-breaking scales.

\subsection{Anomalous $h(125)$ couplings}

In addition to these modifications of the $h$ couplings measured in Higgs production and decay, integrating out the heavy scalars can also induce anomalous couplings of the Higgs 

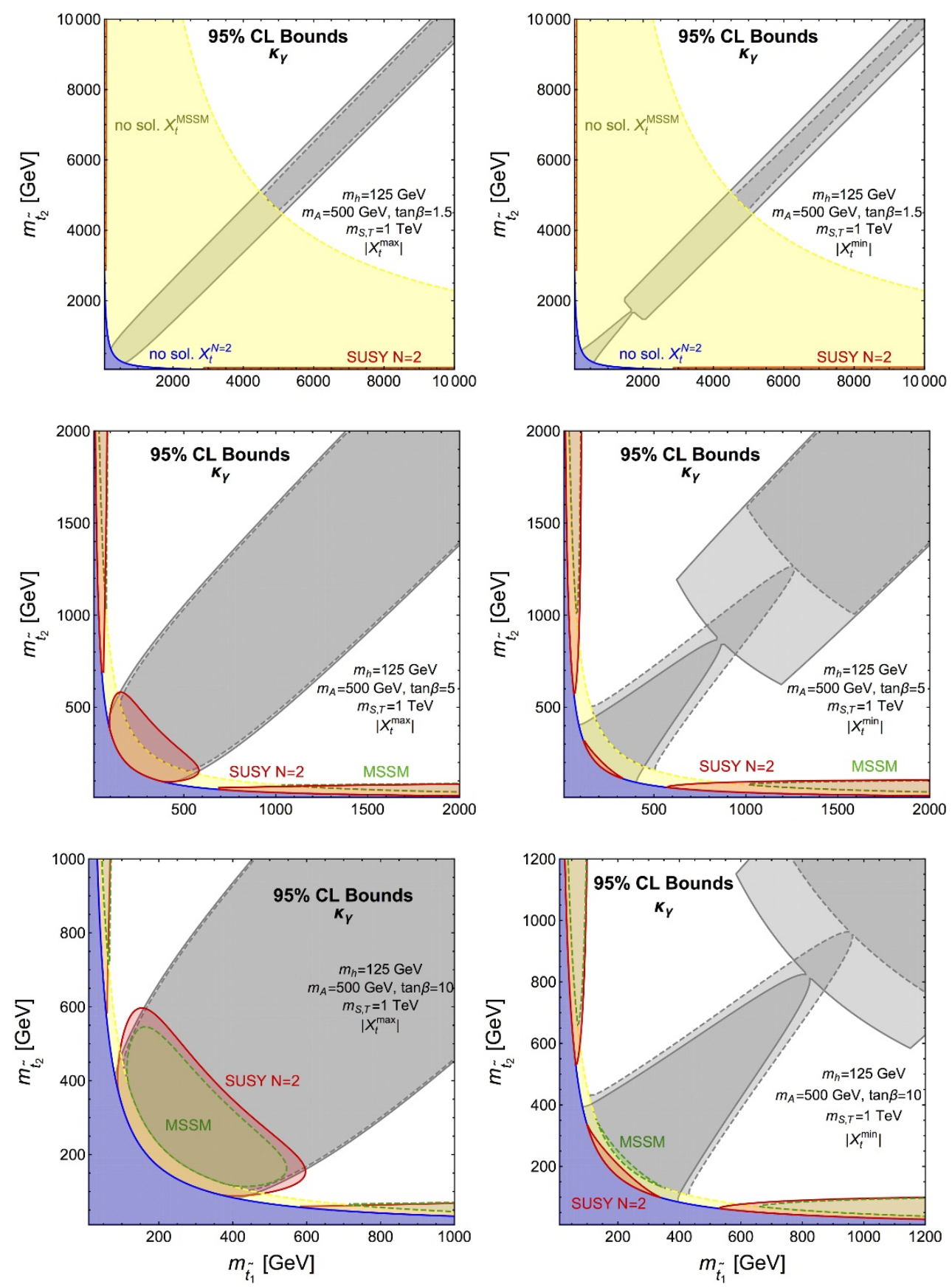

Figure 7. Compilation of the constraints in $\left(m_{\tilde{t}_{1}}, m_{\tilde{t}_{2}}\right)$ planes fixing $X_{t}$ so as to obtain $m_{h}=125 \mathrm{GeV}$ in the $h \mathrm{MSSM}$ and $h 2 \mathrm{MSSM}$, assuming $M_{A}=500 \mathrm{GeV}$ and $\tan \beta=1.5$ (top panels), $\tan \beta=5$ (middle panels) and $\tan \beta=10$ (bottom panels). In the case of the h2MSSM we assume $m_{S}=m_{T}=1 \mathrm{TeV}$. For any given pair of stop masses, the $m_{h}=125 \mathrm{GeV}$ requirement allows at most two solutions for the stop mass mixing parameter, $X_{t}^{2}$. The left (right) panels correspond to the maximal (minimal) solution, $\left|X_{t}^{\max }\right|\left(\left|X_{t}^{\min }\right|\right)$. The grey regions bounded by dashed (full) contours are disallowed by the mixing hypothesis in the $h$ MSSM $(h 2 \mathrm{MSSM})$. Regions where there are no values of $X_{t}$ that yield $m_{h}=125 \mathrm{GeV}$ in the hMSSM (h2MSSM) are shaded yellow (blue). The regions inside the red (green) contours are forbidden by the LHC $h \rightarrow \gamma \gamma$ constraint in the $h 2$ MSSM ( $h$ MSSM). 

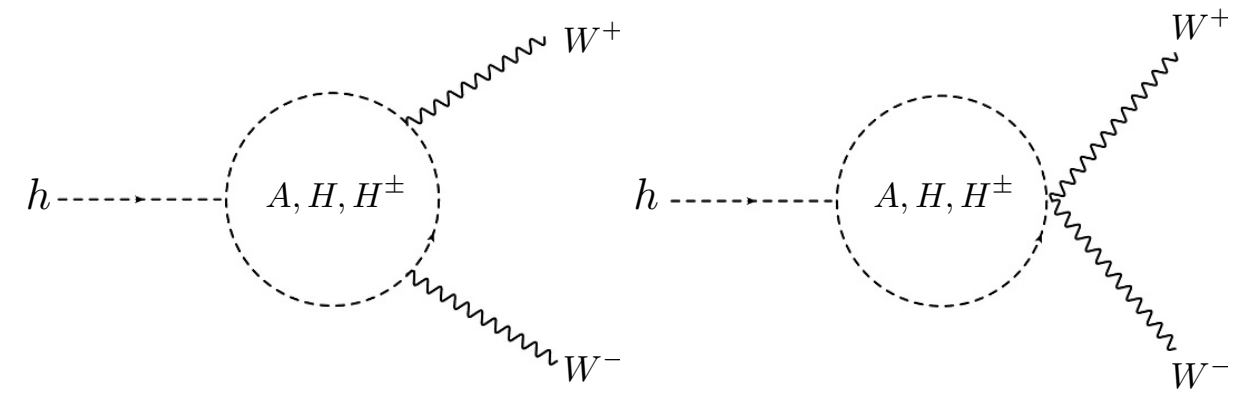

Figure 8. Loop contributions of the heavy scalars to anomalous $h(125)$ couplings.

to vector bosons with non-standard momentum dependence. One can parametrize these effects in the coupling of the Higgs to two $W$ bosons as follows [57]:

$$
\Delta \mathcal{L}_{W}=-\frac{g_{h W W}^{(1)}}{2} W^{\mu \nu} W_{\mu \nu}^{\dagger} h-\left[g_{h W W}^{(2)} W^{\nu} \partial^{\mu} W_{\mu \nu}^{\dagger} h+\text { h.c. }\right]+g_{h W W}^{(3)} W^{\mu} W_{\mu}^{\dagger} h .
$$

We note that the coupling $g^{(3)}$ causes a shift in the usual Standard Model coupling structure. Indeed, the interpretation of the Higgs data described by the Lagrangian (3.2) corresponds to $g^{(3)}=\left(\kappa_{V}-1\right) g_{h W W}$ and setting $g^{(1,2)}$ to zero. However, with more precise measurements of differential distributions in Run 2 one may be able to disentangle different Lorentz structures, which could give a handle for discriminating between an anomaly due to the MSSM and an underlying $N=2$ supersymmetric structure.

Generic expressions for the effects of one-loop scalar contributions to Higgs anomalous couplings can be found in [58]. These correspond to integrating out the heavy MSSM Higgs bosons $A, H$ and $H^{ \pm}$in loops, as shown in figure 8. It is important to note that electroweak precision tests, particularly the constraints from the $S$ and $T$ parameters, require the values of $m_{A}, m_{H}$ and $m_{H^{ \pm}}$to be relatively close to each other. The stops, adjoint scalars, charginos and neutralinos are supposed to be sufficiently massive in our analysis such that their contributions to the electroweak precision parameters are negligable [59-61]. In particular, in a $2 \mathrm{HDM}$ the expression for $\Delta S$ and $\Delta T$ is given by [58]

$$
\begin{aligned}
& \Delta S=-\frac{g_{2}^{2} s_{W}^{2}\left(1-x_{A}+1-x_{0}\right)}{96 \pi^{2} \alpha_{\mathrm{EM}}}, \\
& \Delta T=\frac{m_{H^{ \pm}}^{2}\left(1-x_{A}\right)\left(1-x_{0}\right)}{48 \pi^{2} v^{2} \alpha_{\mathrm{EM}}},
\end{aligned}
$$

where we define the splittings among the heavy scalars by the quantities $x_{0, A}$ :

$$
x_{0} \equiv \frac{m_{H}^{2}}{m_{H^{ \pm}}^{2}}, x_{A} \equiv \frac{m_{A}^{2}}{m_{H^{ \pm}}^{2}} .
$$

and have expanded at linear order in $1-x_{0, A}$. As the splittings in this model are small, imposing the current best fit values from the global analysis of the GFitter group [62] does 
not restrict further the parameter space of $\left(m_{A}, \tan \beta\right)$ from the Higgs coupling constraints. Indeed, $\Delta S, \Delta T \sim 10^{-2}$ for $m_{A} \gtrsim 100 \mathrm{GeV}$.

In this approximation, one can find compact expressions for the anomalous Higgs couplings:

$$
\begin{aligned}
& g_{h W W}^{(1)}=\frac{-g_{2}^{2} v}{192 \pi^{2} m_{H^{ \pm}}^{2}}\left[\frac{g_{0}+g_{A}+2 g_{+}}{2}+\left(1-x_{0}\right) \frac{4 g_{0}+g_{+}}{10}+\left(1-x_{A}\right) \frac{4 g_{A}+g_{+}}{10}\right] \\
& g_{h W W}^{(2)}=\frac{g_{2}^{2} v}{192 \pi^{2} m_{H^{ \pm}}^{2}}\left[\left(1-x_{0}\right) \frac{g_{0}-g_{+}}{20}+\left(1-x_{A}\right) \frac{g_{A}-g_{+}}{20}\right] \\
& g_{h W W}^{(3)}=\frac{g_{2}^{2} v}{192 \pi^{2}}\left[\left(1-x_{0}\right)\left(g_{+}-g_{0}\right)+\left(1-x_{A}\right)\left(g_{+}-g_{A}\right)\right]
\end{aligned}
$$

Here $g_{0, A,+}$ denote the trilinear scalar couplings, $g_{0} \equiv g_{h H H} / v, g_{A} \equiv g_{h A A} / v$ and $g_{+} \equiv$ $g_{h H^{+} H^{-}} / v$. These expressions are generic in a $2 \mathrm{HDM}$ model as long as the expansion in $x_{0, A}$ is justified.

The values of the splittings in the MSSM and its $N=2$ extension can be obtained by inspecting (2.6) and (2.7), respectively. In the $N=2$ case, one finds $x_{0}=x_{A} \simeq 1-m_{W}^{2} / m_{A}^{2}$.

Turning now to the trilinear Higgs couplings, we note that the new $N=2$ term in the scalar potential in (2.3) does not contribute, so the analytical formulae for the trilinear couplings are the same as in the $N=1 \mathrm{MSSM}$, see, e.g., [63]. Therefore, at leading order in $m_{W}^{2} / m_{A}^{2}$, the effect of integrating out the heavy scalars in the $N=2$ extension of the MSSM is to generate anomalous couplings of the Higgs to vector bosons of the type $g_{h W W}^{(1)}$, namely a Higgs coupling to the square of the gauge field strength with magnitude

$$
g_{h W W}^{(1)}=\frac{-g_{2}^{2} v}{192 \pi^{2} m_{A}^{2}}\left[1+2 c_{W}^{2}-3 \frac{m_{h}^{2}-\epsilon}{m_{Z}^{2}}\right] .
$$

Bounds on effective operators in an Effective Field Theory approach from Higgs data using differential distributions $[64,65]$ can be used in our case by noting that the anomalous couplings are related to operators defined there by [58]

$$
\begin{aligned}
g_{h W W}^{(1)} & =\frac{2 g_{2}}{m_{W}} \bar{c}_{H W}, \\
g_{h W W}^{(2)} & =\frac{g_{2}}{2 m_{W}}\left[\bar{c}_{W}+\bar{c}_{H W}\right] .
\end{aligned}
$$

This leads to a specific relation among the operators, namely $\bar{c}_{W}=-\bar{c}_{H W}$ for this model.

A global fit to Higgs and electroweak boson properties in this particular case was made in [64], leading to a bound from the Run 1 data: $\bar{c}_{H W} \in(0.0004,0.02)$, which places no useful constraint on $m_{A}$ currently, as compared with the bounds on total rates discussed before. However, this situation may change with the advent of Run 2 and subsequent Higgs data.

\section{Conclusions}

As discussed in the Introduction, whereas the chiral structure of the Standard Model prevents it from accommodating any more than $N=1$ supersymmetry, any extension of 
the Standard Model at the TeV scale would contain vector-like fermions, and hence could accommodate $N=2$ supersymmetry. A first window on this doubling up of supersymmetry could be provided by the Higgs sector. The two Higgs supermultiplets of the MSSM form a vector-like pair, and thus could accommodate $N=2$ supersymmetry. Measurements of the $h(125)$ boson and searches for heavier Higgs bosons in LHC Run 1 can already be used to probe this possibility.

In order to analyze this option, we have introduced an $h 2 \mathrm{MSSM}$ scenario in which the stop sector is assumed to lift the $h$ mass from its tree-level value to the measured $m_{h}=$ $125 \mathrm{GeV}$ through one-loop radiative corrections. This scenario is exactly analogous to the $h \mathrm{MSSM}$ scenario proposed previously within the usual $N=1$ MSSM context [42-45]. An interesting aspect of the $h 2 \mathrm{MSSM}$ scenario is that much smaller stop masses are required to obtain $m_{h}=125 \mathrm{GeV}$ than are needed in the $h \mathrm{MSSM}$, for any given values of $m_{A}$ and $\tan \beta$.

Another interesting feature of the $N=2$ extension of the MSSM is that the heavy Higgs bosons $H, A, H^{ \pm}$decouple from the massive vector bosons $W^{ \pm}, Z^{0}$ at the tree level. This observation is subject to radiative corrections, but the decoupling limit is a sufficiently good approximation that current searches for $H \rightarrow W^{+} W^{-}, Z^{0} Z^{0}$ and $A \rightarrow Z h$ do not constrain the $h 2 \mathrm{MSSM}$ significantly. On the other hand, the constraints from the decays of the heavy Higgs bosons to fermions are the same in the $h 2 \mathrm{MSSM}$ as in the $h$ MSSM.

The most stringent constraints on the h2MSSM come from LHC Run 1 measurements of the $h(125)$ couplings, including those to fermions, massive and massless gauge bosons. However, these constraints are considerably weaker than in the $h \mathrm{MSSM}$. We find that $m_{A} \gtrsim$ $185 \mathrm{GeV}$ is possible in the $h 2 \mathrm{MSSM}$, whereas $m_{A} \gtrsim 350 \mathrm{GeV}$ is required in the $h \mathrm{MSSM}$.

Looking to the future, we have also calculated the possible $N=2$ Higgs sector contributions to anomalous couplings of the $h(125)$ boson. Current limits on these couplings do not constrain the $N=2$ model, but this may be an interesting window for future measurements at the LHC and elsewhere.

Doubling up supersymmetry opens up the possibility that supersymmetric Higgs bosons and stop squarks could be significantly lighter than in the MSSM. Maybe Run 2 of the LHC will discover not just one supersymmetry, but two?

\section{Acknowledgments}

The work of JE and JQ is supported partly by STFC Grant ST/L000326/1, and that of VS by STFC Grant ST/J000477/1. JE thanks the CERN Theoretical Physics Department for its hospitality, and VS thanks Martin Gorbahn for useful discussions.

Open Access. This article is distributed under the terms of the Creative Commons Attribution License (CC-BY 4.0), which permits any use, distribution and reproduction in any medium, provided the original author(s) and source are credited. 


\section{References}

[1] I. Antoniadis, K. Benakli, A. Delgado, M. Quirós and M. Tuckmantel, Split extended supersymmetry from intersecting branes, Nucl. Phys. B 744 (2006) 156 [hep-th/0601003] [INSPIRE].

[2] I. Antoniadis, K. Benakli, A. Delgado and M. Quirós, A new gauge mediation theory, Adv. Stud. Theor. Phys. 2 (2008) 645 [hep-ph/0610265] [InSPIRE].

[3] I. Antoniadis, A. Delgado, K. Benakli, M. Quirós and M. Tuckmantel, Splitting extended supersymmetry, Phys. Lett. B 634 (2006) 302 [hep-ph/0507192] [InSPIRE].

[4] L. Álvarez-Gaumé and S.F. Hassan, Introduction to $S$ duality in $N=2$ supersymmetric gauge theories: a pedagogical review of the work of Seiberg and Witten, Fortsch. Phys. 45 (1997) 159 [hep-th/9701069] [INSPIRE].

[5] I. Antoniadis, S. Dimopoulos, A. Pomarol and M. Quirós, Soft masses in theories with supersymmetry breaking by TeV compactification, Nucl. Phys. B 544 (1999) 503 [hep-ph/9810410] [INSPIRE].

[6] R. Barbieri, L.J. Hall and Y. Nomura, Models of Scherk-Schwarz symmetry breaking in 5D: classification and calculability, Nucl. Phys. B 624 (2002) 63 [hep-th/0107004] [InSPIRE].

[7] T.-J. Li, $N=2$ supersymmetric GUT breaking on $T^{2}$ orbifolds, Nucl. Phys. B 619 (2001) 75 [hep-ph/0108120] [INSPIRE].

[8] F. del Aguila, M. Dugan, B. Grinstein, L.J. Hall, G.G. Ross and P.C. West, Low-energy models with two supersymmetries, Nucl. Phys. B 250 (1985) 225 [InSPIRE].

[9] N. Polonsky and S.-F. Su, Low-energy limits of theories with two supersymmetries, Phys. Rev. D 63 (2001) 035007 [hep-ph/0006174] [INSPIRE].

[10] P.J. Fox, A.E. Nelson and N. Weiner, Dirac gaugino masses and supersoft supersymmetry breaking, JHEP 08 (2002) 035 [hep-ph/0206096] [INSPIRE].

[11] G.D. Kribs, E. Poppitz and N. Weiner, Flavor in supersymmetry with an extended R-symmetry, Phys. Rev. D 78 (2008) 055010 [arXiv:0712.2039] [INSPIRE].

[12] K. Benakli and M.D. Goodsell, Dirac gauginos in general gauge mediation, Nucl. Phys. B 816 (2009) 185 [arXiv:0811.4409] [InSPIRE].

[13] S.Y. Choi, M. Drees, J. Kalinowski, J.M. Kim, E. Popenda and P.M. Zerwas, Color-octet scalars of $N=2$ supersymmetry at the LHC, Phys. Lett. B 672 (2009) 246 [arXiv: 0812.3586] [INSPIRE].

[14] G. Bélanger, K. Benakli, M. Goodsell, C. Moura and A. Pukhov, Dark matter with Dirac and Majorana gaugino masses, JCAP 08 (2009) 027 [arXiv:0905.1043] [INSPIRE].

[15] K. Benakli and M.D. Goodsell, Dirac gauginos and kinetic mixing, Nucl. Phys. B 830 (2010) 315 [arXiv:0909.0017] [INSPIRE].

[16] K. Benakli and M.D. Goodsell, Dirac gauginos, gauge mediation and unification, Nucl. Phys. B 840 (2010) 1 [arXiv:1003.4957] [InSPIRE].

[17] S.Y. Choi, D. Choudhury, A. Freitas, J. Kalinowski, J.M. Kim and P.M. Zerwas, Dirac neutralinos and electroweak scalar bosons of $N=1 / N=2$ hybrid supersymmetry at colliders, JHEP 08 (2010) 025 [arXiv: 1005.0818] [INSPIRE]. 
[18] S.Y. Choi, D. Choudhury, A. Freitas, J. Kalinowski and P.M. Zerwas, The extended Higgs system in R-symmetric supersymmetry theories, Phys. Lett. B 697 (2011) 215 [Erratum ibid. B 698 (2011) 457] [arXiv: 1012.2688] [INSPIRE].

[19] S. Abel and M. Goodsell, Easy Dirac gauginos, JHEP 06 (2011) 064 [arXiv:1102.0014] [INSPIRE].

[20] K. Benakli, M.D. Goodsell and A.-K. Maier, Generating $\mu$ and B $\mu$ in models with Dirac gauginos, Nucl. Phys. B 851 (2011) 445 [arXiv:1104.2695] [INSPIRE].

[21] H. Itoyama and N. Maru, D-term dynamical supersymmetry breaking generating split $N=2$ gaugino masses of mixed Majorana-Dirac type, Int. J. Mod. Phys. A 27 (2012) 1250159 [arXiv: 1109.2276] [INSPIRE].

[22] M. Heikinheimo, M. Kellerstein and V. Sanz, How many supersymmetries?, JHEP 04 (2012) 043 [arXiv: 1111.4322] [INSPIRE].

[23] G.D. Kribs and A. Martin, Supersoft supersymmetry is super-safe, Phys. Rev. D 85 (2012) 115014 [arXiv: 1203.4821] [INSPIRE].

[24] M.D. Goodsell, Two-loop RGEs with Dirac gaugino masses, JHEP 01 (2013) 066 [arXiv:1206.6697] [INSPIRE].

[25] K. Benakli, M.D. Goodsell and F. Staub, Dirac gauginos and the $125 \mathrm{GeV}$ Higgs, JHEP 06 (2013) 073 [arXiv: 1211.0552] [INSPIRE].

[26] H. Itoyama and N. Maru, D-term triggered dynamical supersymmetry breaking, Phys. Rev. D 88 (2013) 025012 [arXiv:1301.7548] [INSPIRE].

[27] E. Dudas, M. Goodsell, L. Heurtier and P. Tziveloglou, Flavour models with Dirac and fake gluinos, Nucl. Phys. B 884 (2014) 632 [arXiv:1312.2011] [InSPIRE].

[28] H. Itoyama and N. Maru, $126 \mathrm{GeV}$ Higgs boson associated with D-term triggered dynamical supersymmetry breaking, Symmetry 7 (2015) 193 [arXiv:1312.4157] [INSPIRE].

[29] K. Benakli, M. Goodsell, F. Staub and W. Porod, Constrained minimal Dirac gaugino supersymmetric Standard Model, Phys. Rev. D 90 (2014) 045017 [arXiv:1403.5122] [INSPIRE].

[30] M.D. Goodsell and P. Tziveloglou, Dirac gauginos in low scale supersymmetry breaking, Nucl. Phys. B 889 (2014) 650 [arXiv:1407.5076] [InSPIRE].

[31] P. Diessner, J. Kalinowski, W. Kotlarski and D. Stöckinger, Higgs boson mass and electroweak observables in the MRSSM, JHEP 12 (2014) 124 [arXiv:1410.4791] [INSPIRE].

[32] P. Diessner, J. Kalinowski, W. Kotlarski and D. Stöckinger, Two-loop correction to the Higgs boson mass in the MRSSM, Adv. High Energy Phys. 2015 (2015) 760729 [arXiv:1504.05386] [INSPIRE].

[33] M.D. Goodsell, M.E. Krauss, T. Müller, W. Porod and F. Staub, Dark matter scenarios in a constrained model with Dirac gauginos, JHEP 10 (2015) 132 [arXiv:1507.01010] [INSPIRE].

[34] P. Diessner, J. Kalinowski, W. Kotlarski and D. Stöckinger, Exploring the Higgs sector of the MRSSM with a light scalar, JHEP 03 (2016) 007 [arXiv: 1511.09334] [INSPIRE].

[35] J. Braathen, M.D. Goodsell and P. Slavich, Leading two-loop corrections to the Higgs boson masses in SUSY models with Dirac gauginos, JHEP 09 (2016) 045 [arXiv:1606.09213] [INSPIRE]. 
[36] A.E. Nelson, N. Rius, V. Sanz and M. Ünsal, The minimal supersymmetric model without a u term, JHEP 08 (2002) 039 [hep-ph/0206102] [INSPIRE].

[37] ATLAS and CMS collaborations, Combined measurement of the Higgs boson mass in pp collisions at $\sqrt{s}=7$ and $8 \mathrm{TeV}$ with the ATLAS and CMS experiments, Phys. Rev. Lett. 114 (2015) 191803 [arXiv: 1503.07589] [INSPIRE].

[38] K. Benakli, M.D. Goodsell and F. Staub, Dirac gauginos and the 125 GeV Higgs, JHEP 06 (2013) 073 [arXiv: 1211.0552] [inSPIRE].

[39] L.J. Hall and L. Randall, U(1) $)_{R}$ symmetric supersymmetry, Nucl. Phys. B 352 (1991) 289 [INSPIRE].

[40] L. Randall and N. Rius, The minimal $\mathrm{U}(1)_{R}$ symmetric model revisited, Phys. Lett. B 286 (1992) 299 [INSPIRE].

[41] M. Dine and D. MacIntire, Supersymmetry, naturalness and dynamical supersymmetry breaking, Phys. Rev. D 46 (1992) 2594 [hep-ph/9205227] [INSPIRE].

[42] L. Maiani, A.D. Polosa and V. Riquer, Bounds to the Higgs sector masses in minimal supersymmetry from LHC data, Phys. Lett. B 724 (2013) 274 [arXiv:1305.2172] [INSPIRE].

[43] A. Djouadi and J. Quevillon, The MSSM Higgs sector at a high $M_{S U S Y}$ : reopening the low $\tan \beta$ regime and heavy Higgs searches, JHEP 10 (2013) 028 [arXiv: 1304.1787] [INSPIRE].

[44] A. Djouadi, L. Maiani, G. Moreau, A. Polosa, J. Quevillon and V. Riquer, The post-Higgs MSSM scenario: habemus MSSM?, Eur. Phys. J. C 73 (2013) 2650 [arXiv:1307.5205] [INSPIRE].

[45] A. Djouadi, L. Maiani, A. Polosa, J. Quevillon and V. Riquer, Fully covering the MSSM Higgs sector at the LHC, JHEP 06 (2015) 168 [arXiv:1502.05653] [INSPIRE].

[46] E. Bagnaschi et al., Benchmark scenarios for low $\tan \beta$ in the MSSM, LHCHXSWG-2015-002, CERN, Geneva Switzerland (2015).

[47] P. Draper, G. Lee and C.E.M. Wagner, Precise estimates of the Higgs mass in heavy supersymmetry, Phys. Rev. D 89 (2014) 055023 [arXiv: 1312.5743] [INSPIRE].

[48] G. Lee and C.E.M. Wagner, Higgs bosons in heavy supersymmetry with an intermediate $m_{A}$, Phys. Rev. D 92 (2015) 075032 [arXiv:1508.00576] [INSPIRE].

[49] K. Benakli, M.D. Goodsell and A.-K. Maier, Generating $\mu$ and B $\mu$ in models with Dirac gauginos, Nucl. Phys. B 851 (2011) 445 [arXiv:1104.2695] [InSPIRE].

[50] I. Jack and D.R.T. Jones, Nonstandard soft supersymmetry breaking, Phys. Lett. B 457 (1999) 101 [hep-ph/9903365] [INSPIRE].

[51] ATLAS collaboration, Constraints on new phenomena via Higgs boson couplings and invisible decays with the ATLAS detector, JHEP 11 (2015) 206 [arXiv:1509.00672] [INSPIRE].

[52] ATLAS and CMS collaborations, Measurements of the Higgs boson production and decay rates and constraints on its couplings from a combined ATLAS and CMS analysis of the LHC pp collision data at $\sqrt{s}=7$ and 8 TeV, JHEP 08 (2016) 045 [arXiv: 1606.02266] [INSPIRE].

[53] J.F. Gunion, H.E. Haber, G.L. Kane and S. Dawson, The Higgs hunter's guide, Front. Phys. 80 (2000) 1 [INSPIRE]. 
[54] A. Djouadi, V. Driesen, W. Hollik and J.I. Illana, The coupling of the lightest SUSY Higgs boson to two photons in the decoupling regime, Eur. Phys. J. C 1 (1998) 149 [hep-ph/9612362] [INSPIRE].

[55] J.R. Espinosa, C. Grojean, V. Sanz and M. Trott, NSUSY fits, JHEP 12 (2012) 077 [arXiv:1207.7355] [INSPIRE].

[56] A. Djouadi, J. Quevillon and R. Vega-Morales, Into the multi-TeV scale with a Higgs golden ratio, Phys. Lett. B 757 (2016) 412 [arXiv: 1509.03913] [INSPIRE].

[57] P. Artoisenet et al., A framework for Higgs characterisation, JHEP 11 (2013) 043 [arXiv: 1306.6464] [INSPIRE].

[58] M. Gorbahn, J.M. No and V. Sanz, Benchmarks for Higgs effective theory: extended Higgs sectors, JHEP 10 (2015) 036 [arXiv:1502.07352] [INSPIRE].

[59] A.E. Nelson, N. Rius, V. Sanz and M. Ünsal, The minimal supersymmetric model without a $\mu$ term, JHEP 08 (2002) 039 [hep-ph/0206102] [INSPIRE].

[60] G. Marandella, C. Schappacher and A. Strumia, Supersymmetry and precision data after LEP2, Nucl. Phys. B 715 (2005) 173 [hep-ph/0502095] [InSPIRE].

[61] H. Beauchesne and T. Gregoire, Electroweak precision measurements in supersymmetric models with a $\mathrm{U}(1)_{R}$ lepton number, JHEP 05 (2014) 051 [arXiv:1402.5403] [INSPIRE].

[62] GFitTer Group collaboration, M. Baak et al., The global electroweak fit at NNLO and prospects for the LHC and ILC, Eur. Phys. J. C 74 (2014) 3046 [arXiv:1407.3792] [INSPIRE].

[63] A. Djouadi, The anatomy of electro-weak symmetry breaking. II. The Higgs bosons in the minimal supersymmetric model, Phys. Rept. 459 (2008) 1 [hep-ph/0503173] [INSPIRE].

[64] J. Ellis, V. Sanz and T. You, The effective Standard Model after LHC run I, JHEP 03 (2015) 157 [arXiv: 1410.7703] [INSPIRE].

[65] J. Ellis, V. Sanz and T. You, Complete Higgs sector constraints on dimension-6 operators, JHEP 07 (2014) 036 [arXiv: 1404.3667] [INSPIRE].

[66] Y. Okada, M. Yamaguchi and T. Yanagida, Upper bound of the lightest Higgs boson mass in the minimal supersymmetric Standard Model, Prog. Theor. Phys. 85 (1991) 1 [INSPIRE].

[67] J.R. Ellis, G. Ridolfi and F. Zwirner, Radiative corrections to the masses of supersymmetric Higgs bosons, Phys. Lett. B 257 (1991) 83 [INSPIRE].

[68] H.E. Haber and R. Hempfling, Can the mass of the lightest Higgs boson of the minimal supersymmetric model be larger than $m_{Z}$ ?, Phys. Rev. Lett. 66 (1991) 1815 [INSPIRE]. 